\title{
Early life and adult stress promote sex dependent changes in hypothalamic miRNAs and environmental enrichment prevents stress-induced miRNA and gene expression changes in rats
}

\author{
Lauren Allen McKibben and Yogesh Dwivedi
}

\begin{abstract}
Background: The hypothalamus plays a key role in the stress response. While early life stress (ELS) increases susceptibility to psychiatric disorders including major depressive disorder (MDD), acute stress during adulthood can also precipitate MDD after ELS.

Aim: Here, we tested the expression of miRNAs following ELS and susceptibility to depression-like behavior and whether sex or acute stress exacerbates this response. We also tested whether environmental enrichment (Enr) promotes early life and adult behavioral stress resilience and its effect on hypothalamic miRNA and gene expression. Following rat maternal separation (MS) as an ELS model, Enr from weaning through adulthood, and restraint (RS) as acute adult stress, we tested both animal behavior and miRNA expression in the hypothalamus. Target genes and their enrichment and ontology were analyzed using bioinformatic tools. Target gene expression changes were tested using $\mathrm{PPCR}$, and miRNA promoter methylation was studied using methylated-DNA immunoprecipitation QPCR.

Results: MS, Enr, RS, and sex altered hypothalamic miRNAs, including several previously reported in MS literature: miRs-29, $-124,-132,-144,-504$. Sex had a significant effect on the greatest number of miRNAs. Also, Enr reversed downregulation of miR-29b-1-5p and -301b-3p in MS. qPCR showed that MAPK6 and MMP19, targets of miR-301b-3p, were upregulated in MS and reversed by Enr. Additionally, miR-219a was hypermethylated in MS coinciding with decreased miR-219a expression.

Conclusions: This study found that sex plays a critical role in the hypothalamic miRNA response to both ELS and acute stress, with males expressing greater changes following postnatal stress. Moreover, enrichment significantly altered behavior as well as hypothalamic miRNA expression and their gene targets. Because of its role as the initiator of the autonomic stress response and connection to hedonic and motivational behavior, the hypothalamic miRNA landscape may significantly alter both the short and long-term behavioral response to stress.
\end{abstract}

\footnotetext{
* Correspondence: ydwivedi@uab.edu

Present address: Department of Psychiatry and Behavioral Neurobiology, University of Alabama at Birmingham, SC711 Sparks Center, 1720 2nd Avenue South, Birmingham, AL 35294, USA
}

C The Author(s). 2021 Open Access This article is licensed under a Creative Commons Attribution 4.0 International License, which permits use, sharing, adaptation, distribution and reproduction in any medium or format, as long as you give appropriate credit to the original author(s) and the source, provide a link to the Creative Commons licence, and indicate if changes were made. The images or other third party material in this article are included in the article's Creative Commons licence, unless indicated otherwise in a credit line to the material. If material is not included in the article's Creative Commons licence and your intended use is not permitted by statutory regulation or exceeds the permitted use, you will need to obtain permission directly from the copyright holder. To view a copy of this licence, visit http://creativecommons.org/licenses/by/4.0/. The Creative Commons Public Domain Dedication waiver (http://creativecommons.org/publicdomain/zero/1.0/) applies to the data made available in this article, unless otherwise stated in a credit line to the data. 
Keywords: Early life stress, Maternal separation, microRNA, Hypothalamus, Restraint stress, Enrichment, microRNA sequencing, Methylation, Rat

\section{Background}

Early life stress (ELS), namely abuse, neglect, and household dysfunction, is estimated to affect $64 \%$ of the US population [1]. This poses a major public health risk as ELS increases susceptibility to psychiatric disorders, including major depressive disorder (MDD). Not only does MDD carry a sizeable economic burden due to lost productivity [2], its symptoms are particularly debilitating. MDD onset has also been strongly associated with a precipitating stressful event, especially within the previous month [3]. Moreover, ELS and stress during adulthood can interact producing more severe or long-lasting symptoms than individually [4], also described as the two/three-hit hypothesis of stress susceptibility [5]. There are also significant sex differences in the response to ELS [6]. In the US, men and women experience similar levels of ELS [7], yet women are twice as likely to develop MDD [8]. There are only a limited number of studies that have examined sex-mediated depressive response to ELS [9].

Previous preclinical and clinical studies have implicated hypothalamic-pituitary-adrenal (HPA)-axis responsiveness in the depressive effects of ELS [10-12]. The HPA axis is the primary neurochemical stress response system whereby corticotropin releasing hormone (CRH) is secreted from the hypothalamus triggering a cascade of hormone release by the pituitary (adrenocorticotropin-releasing hormone-ACTH) and adrenal glands (corticosterone-CORT). Not only is the hypothalamus the initial site of this neuroendocrine response, but it also receives inputs from regions central to emotion processing like the amygdala [13] and frontal cortex [14]. Moreover, it plays a particularly important role in the symptomatology of MDD [15]. The hypothalamus receives sensory cues predicting reward (or lack thereof) and stimulates dopamine downstream [16]. In rodents, disruption of dopaminergic projections to the hypothalamus leads to reduced sucrose preference (i.e. more anhedonia-like behavior) [17]. Similarly, antagonizing hypothalamic GABA receptors increases anxiety-related measures in the elevated plus maze (EPM) such as time spent and number of entries into the closed arms [18] and directly injecting calcium channel inhibitors into the hypothalamus increases swimming behavior in the forced swim test (FST) indicated reduced behavioral despair [19]. These findings point to several neurochemical signaling mechanisms in the hypothalamus which might contribute to increased stress and depression-related behaviors. ELS has also been associated with disturbed
HPA function including increased cortisol response to ACTH administration and increased cerebrospinal fluid CRH levels in MDD patients [20, 21]. While our understanding of the HPA stress response and its interaction with ELS has improved over the decades, currently available treatments for MDD are only modestly effective.

Recently, microRNAs (miRNAs) have been proposed as potential therapeutic or biomarker targets for MDD [2225]. These small non-coding RNAs ( 22 nucleotides) are synthesized in the cell nucleus as a hairpin loop and then exported into the cytoplasm where the loop is cleaved. This mature, single-stranded miRNA is loaded into the RNA-induced silencing complex (RISC) and targets the 3'UTR of genes with partially matching nucleotide sequences [26]. Canonically, miRNAs block the translation of mRNAs into proteins. Furthermore, because miRNAs are relatively short and can bind to mRNAs without perfect base pairing, they are able to target many different genes concurrently. Only a few studies to date have explored the effect of ELS on miRNAs (reviewed in [27]), one of which employed short duration maternal separation (MS) as a form of enrichment and found increases in miRs-488, -144 , and $-542-5 p$ and decreases in miRs421 and -376b-5 in hypothalamus [28]. MS has been well established as a rodent model of ELS associated with depression- and anxiety-like behavior [29]. MS protocols vary, but typically rodent pups are separated from their dam for $180 \mathrm{~min}$ daily for 12-21 days. Another study combined postnatal MS and restraint stress (RS) during adulthood to test if RS precipitated depressive behavior after MS [30]. Animals who experienced both MS and RS showed increased immobility in the forced swim test and decreased sucrose preference along with increased medial prefrontal cortex expression of miR-124 [30]. Increased miR-124 in the dentate gyrus after $90 \mathrm{~min}$ MS has also been reported [31]. While miRNAs serve a primary function to regulate gene expression, they are also regulated by epigenetic modifications such as methylation [32]. Only a few studies have elucidated miRNA methylation changes in psychiatric disorders. In adolescent patients with high risk for MDD, significant hypomethylation was found in miR-4646-3p promoter region [33]. Using chronic CORT administration to induce depression-like behavior in rats, our group found promoter hypomethylation of miR-124$3 p$ [34]. No studies have explored miRNA promoter methylation in an ELS model.

There has been a growing shift toward nonpharmaceutical treatments in mental health. Clinical trials have increasingly pursued behavioral interventions while 
pharmaceutical-based trials have decreased from $43 \%$ in 2007 to $27 \%$ by 2018 [35]. Depressed individuals with a history of ELS may respond less to antidepressant drug therapy than patients with no ELS history [36]; however, very little is yet understood about how nonpharmaceutical methods contribute to healthy brain function. Environmental enrichment (Enr) has been found to improve behaviors in rodents related to anxiety $[37,38]$ and MDD [39] and lower blood CORT levels after RS [38]. Earlier work proposed that Enr reverses HPA dysregulation after ELS [40, 41].

The purpose of the study was: 1) to show whether ELS-induced depressive behavior is associated with miRNA expression changes in the hypothalamus and whether there is an interaction between MS, RS, and sex on miRNA expression; 2) to examine the effect of the interaction between RS, sex and Enr in MS animals on hypothalamic miRNA expression; 3) explore if these MS-induced miRNAs changes in the hypothalamus relate to gene expression changes and whether these miRNAs themselves might be regulated via methylation. To examine these, we assessed the behavioral and physiological consequences of MS and Enr as well as genomewide changes in miRNA expression using nextgeneration sequencing. We also used bioinformatic tools to understand the potential functions associated with altered miRNAs and their targets. Finally, we tested the expression of stress-related miRNA gene targets and potential regulation of miRNAs by promoter region methylation.

\section{Results}

\section{Differential miRNA expression in MS, RS, and enriched} animals

Animals were assigned to MS, RS, and Enr groups or relative controls. As shown in Fig. 1a MS animals were separated for 14 days and enrichment began immediately post-weaning; control animals were handled daily but not separated and were housed in standard conditions. Animals in RS groups were restrained for 7 days prior to behavioral testing and tissue collection; animals assigned to non-RS control groups were briefly handled daily. Finally, RNA isolated from the hypothalamus was sequenced to determine miRNA expression differences across these groups; animal groups for each set of comparisons are shown in Fig. 1b.

\section{Behavior differences resulting from MS, RS, and sex}

We tested for main and interaction effects on physiological and behavioral measures of stress (animals included in this analysis are noted in Fig. 1b by the label "MS x RS x sex") and detected a significant 3-way interaction between MS, RS and sex on EPM closed arm time $(\mathrm{F}(1,48)=5.30, p=0.027$; Fig. 1c), however there was no significant effect on the EPM index score $(p>0.05$; Fig. 1d). Follow-up pairwise t-tests showed that RS-only males spent more time in the closed arms compared to $\mathrm{MS}, \mathrm{MS}+\mathrm{RS}$, and controls $(\mathrm{t}(22)=-2.423, p=0.056)$. There was a significant decrease in sucrose preference for all MS animals compared to all non-MS $(F(1,48)=$ 9.24, $p=0.004$;Fig. 1e). RS significantly altered the effect of sex on body weight $(\mathrm{F}(1,48)=5.79, p=0.021$;Fig. 1f $)$. There was also a significant main effect of sex on escape latency $(\mathrm{F}(1,48)=7.70, p=0.008$;Fig. $1 \mathrm{~g})$ with all males exhibiting higher escape latency than females. We found significantly decreased total movement in the EPM in all female animals compared to males $(\mathrm{F}(1,48)=9.20, p=$ 0.004;Fig. 1h). We also found that forced swim test (FST) swim scores were higher in female than male animals $(F(1,48)=4.19, p=0.047$;Fig. 1i) but climb scores were higher in males than females $(\mathrm{F}(1,48)=8.61, p=$ 0.006;Fig. 1j); there were no differences in FST float score.

\section{The interaction of MS, RS, and sex on corticosterone levels}

We compared plasma CORT level across groups using an independent samples t-tests (Fig. 1k) but there were no significant differences $(p>0.05)$. In males, RS significantly increased CORT level compared to controls and MS-only males $(\mathrm{t}(22)=-2.511, p<.05)$.

\section{The effect of estrus on behavior and miRNAs}

Because of the significant sex differences in behavior and concerns for the effect of female sex hormones on stress-related changes, we tested female animals for estrus phase via vaginal lavage. Representative microscope images of cell cytology and group behavior differences are shown in Supplementary Fig. 1. Because there were no significant behavior differences between the estrus phase groups further analyses did not include this variable. Detailed results are described in Supplementary Table 5.

\section{Behavior differences resulting from RS, enrichment, and sex in MS rats}

A RS $x$ Enr $x$ Sex ANOVA was conducted to identify the effect of these factors and their interaction on behavior. For this analysis, only MS animals were considered because we were primarily interested in exploring enrichment as a preventative measure as opposed to its use simply as enrichment in normal controls (animals included in this analysis are noted in Fig. $1 \mathrm{~b}$ by the label "RS x Enr x sex").

There were no significant effects of MS, RS, or Enr on EPM closed arm frequency (Fig. 1l) or the anxiety index (Fig. $1 \mathrm{~m}$ ). Enr significantly increased total movement in the EPM, regardless of $\mathrm{RS}(\mathrm{F}(1,48)=22.91, p<0.000$;Fig. 1n). All Enr animals regardless of RS showed a 


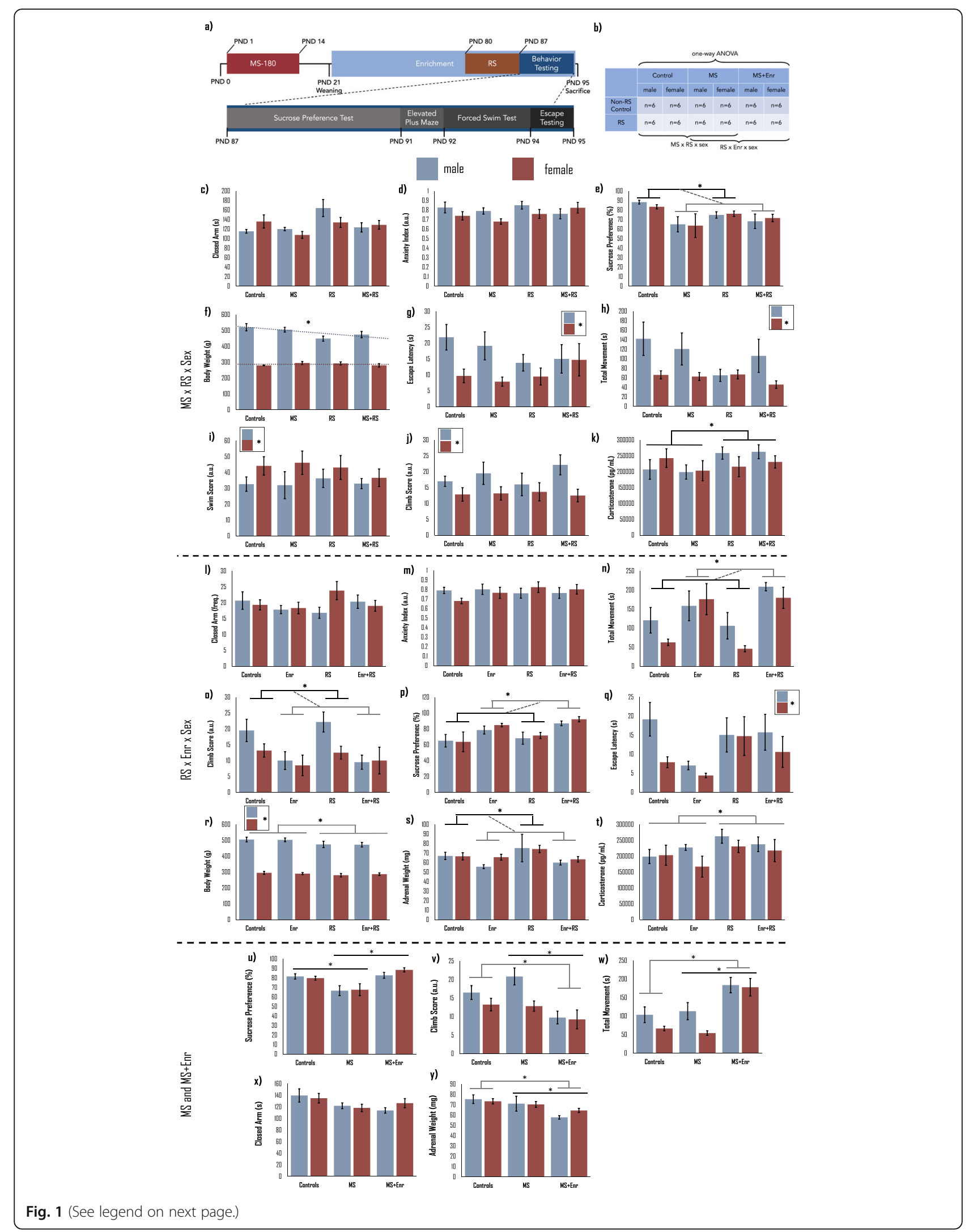


(See figure on previous page.)

Fig. 1 Behavior and physiology associated with maternal separation, restraint stress, sex, and enrichment. A schematic diagram (a) shows the timeline of behavioral testing relative to MS, Enr, and RS. MS was conducted for 180 min daily for the first 14 postnatal days. Enr was provided to animals following weaning until tissues were harvested at PND 95. b) Statistical comparisons of behavior and miRNA expression were conducted using 3-way ANOVA across MS, RS, and sex ( $n=6$ animals per group; first 4 columns of Fig. 1b). Another 3-way analysis was conducted across RS, Enr, and sex, only in MS animals (last 4 columns of Fig. 1b). Analysis of Enr as a treatment following MS was conducted using one-way ANOVA with control, MS, and MS + Enr as groups (indicated across the top of Fig. 1b; $n=12$ animals per group including $n=6$ RS and $n=6$ Non-RS animals per group). c-k) Behavioral changes based on the interaction between MS, RS, and sex are shown. Importantly, MS reduced sucrose preference $(\mathbf{e} ; \mathrm{F}(1,48)=9.24, p=0.004)$ regardless of RS or sex. Sex was a significant predictor for escape latency $(\mathbf{g} ; \mathrm{F}(1,48)=7.70, p=0.008)$, EPM movement (h; $F(1,48)=9.20, p=0.004)$, and FST swim and climb scores (i; $F(1,48)=4.19, p=0.047 ; \mathbf{j} ; F(1,48)=8.61, p=0.006)$. I-t) In MS animals, a 3way ANOVA revealed increased EPM movement $(\mathbf{n} ; \mathrm{F}(1,48)=22.91, p<0.000)$ and sucrose preference $(\mathbf{p}:(1,48)=16.05, p<0.000)$ and decreased FST climbing (o; $F(1,48)=11.92, p=0.001)$ and adrenal weight $(\mathbf{s} ; F(1,48)=5.23, p=0.028)$ following Enr. RS significantly increased plasma Cort levels (t; $\mathrm{F}(1,48)=4.39, p<0.05)$. One-way ANOVA comparison of control, MS, and MS + Enr groups $(\mathbf{u}-\mathbf{y})$ revealed the Enr recovered sucrose preference changes induced by MS $(\mathbf{u} ; F(2,69)=12.049, p<.001)$ as well as recovering FST climbing changes in male animals $(\mathbf{v} ; F(2,69)=6.841, p<0.005)$. Bars represent Mean \pm SEM with males in blue and females in red. Dotted linear trend lines in $\mathbf{f}$ indicate an interaction. Significance: ${ }^{*} p<0.05$. Abbreviations: PND-postnatal day, MS-maternal separation, RS-restraint stress, Enr-environmental enrichment

significant reduction in climb scores compared to nonenriched animals $(\mathrm{F}(1,48)=11.92, p=0.001$;Fig. 1o). Enrichment significantly increased sucrose preference in MS animals regardless of whether they had received RS $(\mathrm{F}(1,48)=16.05, p<0.000$;Fig. 1p). There were no significant effects on escape latency ( $p>0.05$;Fig. 1q). As expected, there was a significant main effect of sex on body weight $(\mathrm{F}(1,48)=495.06, p<0.000)$, but we also found that RS decreased body weight $(\mathrm{F}(1,48)=4.82, p=$ 0.034 ;Fig. 1r) compared to all animals without RS. Adrenal weight was significantly reduced by Enr regardless of RS $(F(1,48)=5.23, p=0.028$;Fig. 1s). Finally, we found that RS increased plasma CORT levels (Fig. 1t; F(1,48)= 4.39, $p<0.05)$.

\section{miRNAs altered by the interaction of MS, RS, and sex}

3-way ANOVA was used to detect significant miRNA expression differences associated with MS, RS, and sex (Table 1). The fold change column headers indicate the two comparison groups and direction of change (e.g. a fold change value of 1.33 for "MS:control" indicates 1.33-times fold change increase in expression in MS relative to control animals). Specific $p$ values are listed alongside fold change in Table 1 . Only miR-26a-3p was significantly affected by the $3-$ way interaction $(p<0.05) .5$ miRNAs were affected by the interaction of MS and RS, 11 by sex and MS, and 46 by sex and RS $(p<0.05)$. Alone, sex significantly altered 24 miRNAs while MS affected 28 and RS affected $43(p<0.05)$. The miRNAs with the greatest fold change were miR-547-3p which was 2.45 -fold downregulated in all females compared to all males $(p<0.05)$, miR-144-3p which was 2.3 -fold downregulated in MS males compared to male controls $(p<0.05)$, and miR-23a-5p which was increased 1.97-fold in all females as compared to males $(p<0.05)$.

miRNAs altered by the interaction of RS, enrichment, and sex in maternally separated animals

miRNAs significantly altered by RS, Enr, sex or their interaction (based on 3-way ANOVA) in MS animals are reported in Table 2 along with their fold change and specific significance ( $p$ value). The 3-way interaction (RS, Enr, and sex) had a significant effect on 32 miRNAs. The largest number of miRNAs were affected by the interaction between Enr and sex, with 56 miRNAs significantly altered. There was a significant interaction between RS and Enr on miR-135b-3p expression $(p<0.05)$. miR-666-3p was significantly affected by the interaction between RS and sex $(p<0.05)$. We detected 2 miRNAs associated with RS main effect: miR-338-5p and -341 $(p<0.05)$. There was a main effect of Enr on 39 miRNAs and sex affected expression of 14 miRNAs. The greatest fold change in expression was found for miR-547-3p with females showing a 2.51-fold downregulation compared to males $(p<0.05)$ regardless of RS or Enr. Next, miR-539-5p showed the next greatest fold change at 1.84-fold increased expression in Enr females compared to non-Enr females $(p<0.05)$.

miRNA-mediated hypothalamic signaling changes after MS and enrichment

Enrichment as prevention of depression- and anxietyrelated behavior after MS

Our primary focus for this study was to elucidate hypothalamic miRNA changes resulting from MS and environmental enrichment. One-way ANOVA was used to compare controls, MS, and MS + Enr animals. Each group (controls, MS, and MS + Enr) consisted of 24 animals (12 male and 12 female) including RS animals; animals included in this analysis are noted in Fig. $1 \mathrm{~b}$ as "one-way ANOVA". There were significant group differences in sucrose preference $(\mathrm{F}(2,69)=12.049, p<.001)$; MS significantly decreased sucrose preference $(p<0.005)$ which was returned to normal levels by Enr $(p<0.001$; Fig. 1u). Climb score (Fig. 1v) in the FST was significantly decreased by Enr as compared to both control $(p<0.05)$ and MS $(p<0.005)$ animals $(\mathrm{F}(2,69)=6.841$, $p<0.005)$. In the EPM, Enr significantly increased total movement (Fig. 1w) compared to controls $(p<0.001)$ 
Table 1 miRNAs altered by the interaction between maternal separation, restraint stress, and sex

\begin{tabular}{|c|c|c|c|c|}
\hline & & CPM-Fold Change & & \\
\hline Accession ID & miRNA & MS: Control & $p$ & Sig. \\
\hline \multicolumn{5}{|l|}{ maternal separation } \\
\hline MIMAT0012855 & miR-666-5p & 1.33 & 0.046 & * \\
\hline MIMAT0017819 & miR-3557-5p & 1.28 & 0.044 & * \\
\hline MIMAT0000838 & miR-132-3p & 1.28 & 0.005 & * \\
\hline MIMAT0003379 & $\operatorname{miR}-378 a-3 p$ & 1.28 & 0.024 & * \\
\hline MIMAT0017158 & miR-212-5p & 1.25 & 0.008 & * \\
\hline MIMAT0003378 & $m i R-378 a-5 p$ & 1.21 & 0.026 & * \\
\hline MIMAT0035730 & miR-1843b-5p & 1.20 & 0.025 & * \\
\hline MIMAT0000859 & miR-181b-5p & 1.19 & 0.020 & * \\
\hline MIMAT0003205 & $m i R-409 a-3 p$ & 1.18 & 0.030 & * \\
\hline MIMAT0000550 & miR-323-3p & 1.16 & 0.024 & * \\
\hline MIMAT0001626 & miR-431 & 1.12 & 0.033 & * \\
\hline MIMAT0003194 & $m i R-376 c-3 p$ & 1.10 & 0.050 & * \\
\hline MIMAT0000832 & miR-126a-3p & 1.09 & 0.028 & * \\
\hline MIMAT0004706 & let-7e-3p & 1.08 & 0.028 & * \\
\hline MIMAT0004732 & $\operatorname{miR}-135 a-3 p$ & -1.11 & 0.046 & * \\
\hline MIMAT0000805 & miR-30e-5p & -1.18 & 0.034 & * \\
\hline MIMAT0004726 & miR-101a-5p & -1.20 & 0.042 & * \\
\hline MIMAT0017026 & miR-301a-5p & -1.20 & 0.046 & * \\
\hline MIMAT0017143 & miR-186-3p & -1.21 & 0.022 & * \\
\hline MIMAT0005328 & miR-673-5p & -1.21 & 0.006 & * \\
\hline MIMAT0035720 & let-7g-3p & -1.23 & 0.031 & * \\
\hline MIMAT0017839 & miR-3065-5p & -1.24 & 0.033 & * \\
\hline MIMAT0000812 & $\operatorname{miR}-33-5 p$ & -1.31 & 0.039 & * \\
\hline MIMAT0017230 & miR-497-3p & -1.39 & 0.024 & * \\
\hline MIMAT0005304 & $m i R-301 b-3 p$ & -1.56 & 0.011 & * \\
\hline MIMAT0005445 & miR-29b-1-5p & -1.71 & 0.012 & * \\
\hline MIMAT0017154 & miR-206-5p & -1.77 & 0.017 & * \\
\hline MIMAT0000850 & miR-144-3p & -1.78 & 0.011 & * \\
\hline restraint stress & & RS:Control & & \\
\hline MIMAT0017881 & miR-3586-3p & 1.26 & 0.016 & * \\
\hline MIMAT0000791 & $\operatorname{miR}-22-3 p$ & 1.21 & 0.014 & * \\
\hline MIMAT0000784 & $\operatorname{miR}-15 b-5 p$ & 1.20 & 0.008 & * \\
\hline MIMAT0003380 & miR-505-3p & 1.20 & 0.011 & * \\
\hline MIMAT0003193 & miR-494-3p & 1.20 & 0.029 & * \\
\hline MIMAT0017840 & $m i R-3065-3 p$ & 1.19 & 0.038 & * \\
\hline MIMAT0003175 & miR-543-3p & 1.17 & 0.038 & * \\
\hline MIMAT0000794 & miR-24-3p & 1.17 & 0.012 & * \\
\hline MIMAT0000792 & $\operatorname{miR}-23 a-3 p$ & 1.17 & 0.034 & * \\
\hline MIMAT0000793 & $\operatorname{miR}-23 b-3 p$ & 1.17 & 0.002 & $* *$ \\
\hline MIMAT0004714 & miR-26b-3p & 1.17 & 0.030 & * \\
\hline MIMAT0035719 & let-7g-5p & 1.13 & 0.040 & * \\
\hline MIMAT0000840 & miR-134-5p & -1.09 & 0.040 & * \\
\hline
\end{tabular}


Table 1 miRNAs altered by the interaction between maternal separation, restraint stress, and sex (Continued)

\begin{tabular}{|c|c|c|c|c|}
\hline & & CPM-Fold Change & & \\
\hline MIMAT0017307 & $m i R-434-5 p$ & -1.10 & 0.047 & * \\
\hline MIMAT0017306 & $\operatorname{miR}-425-3 p$ & -1.10 & 0.016 & * \\
\hline MIMAT0005339 & $\operatorname{miR}-873-5 p$ & -1.11 & 0.037 & * \\
\hline MIMAT0017031 & miR-329-5p & -1.11 & 0.010 & * \\
\hline MIMAT0017137 & $\operatorname{miR}-181 c-3 p$ & -1.13 & 0.040 & * \\
\hline MIMAT0000902 & $\operatorname{miR}-300-3 p$ & -1.13 & 0.023 & * \\
\hline MIMAT0005332 & $\operatorname{miR}-708-3 p$ & -1.14 & 0.043 & * \\
\hline MIMAT0017117 & $\operatorname{miR}-127-5 p$ & -1.14 & 0.009 & * \\
\hline MIMAT0004655 & $\operatorname{miR}-345-3 p$ & -1.14 & 0.015 & * \\
\hline MIMAT0005341 & miR-488-3p & -1.15 & 0.026 & $*$ \\
\hline MIMAT0003192 & $\operatorname{miR}-379-5 p$ & -1.15 & 0.001 & $* * *$ \\
\hline MIMAT0024847 & miR-1843a-5p & -1.15 & 0.030 & * \\
\hline MIMAT0003199 & miR-381-3p & -1.16 & 0.012 & * \\
\hline MIMAT0000781 & $\operatorname{miR}-9 a-5 p$ & -1.17 & 0.016 & * \\
\hline MIMAT0004728 & $\operatorname{miR}-124-5 p$ & -1.18 & 0.027 & * \\
\hline MIMAT0000802 & miR-29a-3p & -1.18 & 0.012 & * \\
\hline MIMAT0004740 & miR-218a-2-3p & -1.19 & 0.012 & * \\
\hline MIMAT0005312 & $\operatorname{miR}-411-5 p$ & -1.20 & 0.002 & $* *$ \\
\hline MIMAT0017334 & $\operatorname{miR}-146 b-3 p$ & -1.20 & 0.010 & * \\
\hline MIMAT0000833 & miR-127-3p & -1.21 & 0.014 & * \\
\hline MIMAT0005308 & miR-380-5p & -1.21 & 0.005 & $* *$ \\
\hline MIMAT0017360 & miR-582-3p & -1.22 & 0.016 & * \\
\hline MIMAT0017828 & miR-3559-3p & -1.22 & 0.018 & * \\
\hline MIMAT0017291 & miR-879-3p & -1.22 & 0.007 & $*$ \\
\hline MIMAT0000587 & miR-341 & -1.23 & 0.004 & $* *$ \\
\hline MIMAT0005325 & $\operatorname{miR}-598-3 p$ & -1.24 & 0.002 & $* *$ \\
\hline MIMAT0001534 & miR-448-3p & -1.27 & 0.024 & * \\
\hline MIMAT0000814 & $m i R-34 c-5 p$ & -1.28 & 0.014 & * \\
\hline MIMAT0024856 & miR-6216 & -1.39 & 0.038 & * \\
\hline MIMAT0017896 & miR-3593-5p & -1.84 & 0.045 & * \\
\hline sex & & Female:Male & & \\
\hline MIMAT0004712 & $\operatorname{miR}-23 a-5 p$ & 1.97 & 0.031 & * \\
\hline MIMAT0004739 & miR-204-3p & 1.58 & 0.027 & * \\
\hline MIMAT0000877 & miR-204-5p & 1.29 & 0.023 & * \\
\hline MIMAT0017802 & miR-3547 & 1.27 & 0.039 & * \\
\hline MIMAT0004729 & $\operatorname{miR}-125 a-3 p$ & 1.21 & 0.045 & * \\
\hline MIMAT0003378 & $\operatorname{miR}-378 a-5 p$ & 1.20 & 0.030 & * \\
\hline MIMAT0001549 & $\operatorname{miR}-365-3 p$ & 1.19 & 0.016 & * \\
\hline MIMAT0017175 & $\operatorname{miR}-421-3 p$ & 1.18 & 0.000 & $* * *$ \\
\hline MIMAT0004648 & miR-339-3p & 1.16 & 0.043 & $*$ \\
\hline MIMAT0004740 & miR-218a-2-3p & 1.15 & 0.041 & * \\
\hline MIMAT0017306 & $\operatorname{miR}-425-3 p$ & 1.11 & 0.011 & * \\
\hline MIMAT0000774 & let-7a-5p & 1.11 & 0.043 & * \\
\hline MIMAT0000796 & $\operatorname{miR}-26 a-5 p$ & 1.09 & 0.006 & * \\
\hline
\end{tabular}


Table 1 miRNAs altered by the interaction between maternal separation, restraint stress, and sex (Continued)

\begin{tabular}{|c|c|c|c|c|c|}
\hline & & CPM-Fold Change & & & \\
\hline MIMAT0005335 & miR-758-3p & -1.11 & & 0.034 & * \\
\hline MIMAT0005299 & miR-181d-5p & -1.12 & & 0.046 & * \\
\hline MIMAT0003207 & $\operatorname{miR}-369-3 p$ & -1.13 & & 0.022 & $*$ \\
\hline MIMAT0017867 & miR-3579 & -1.20 & & 0.039 & * \\
\hline MIMAT0017192 & miR-433-5p & -1.22 & & 0.038 & * \\
\hline MIMAT0005311 & miR-410-3p & -1.27 & & 0.007 & * \\
\hline MIMAT0017136 & miR-154-3p & -1.37 & & 0.022 & * \\
\hline MIMAT0017130 & $\operatorname{miR}-144-5 p$ & -1.49 & & 0.017 & $*$ \\
\hline MIMAT0001633 & $\operatorname{miR}-451-5 p$ & -1.73 & & 0.006 & $*$ \\
\hline MIMAT0000850 & $\operatorname{miR}-144-3 p$ & -1.83 & & 0.008 & * \\
\hline MIMAT0012851 & miR-547-3p & -2.45 & & 0.000 & ** \\
\hline$M S \times R S$ & & MS: Control & $\mathrm{MS}+\mathrm{RS}: \mathrm{RS}$ & & \\
\hline MIMAT0017117 & miR-127-5p & -1.12 & 1.11 & 0.028 & * \\
\hline MIMAT0005325 & miR-598-3p & -1.15 & 1.14 & 0.038 & * \\
\hline MIMAT0017851 & miR-3571 & -1.31 & 1.42 & 0.020 & * \\
\hline MIMAT0017818 & miR-3556b & -1.31 & 1.01 & 0.043 & * \\
\hline MIMAT0001534 & $m i R-448-3 p$ & -1.37 & 1.13 & 0.031 & * \\
\hline MS $x$ sex & & MS Male: Control Male & MS Female: Control Female & & \\
\hline MIMAT0012830 & miR-504 & 1.28 & -1.20 & 0.044 & $*$ \\
\hline MIMAT0005278 & $\operatorname{miR}-466 b-5 p$ & 1.03 & -1.19 & 0.047 & * \\
\hline $\begin{array}{l}\text { MIMAT0000839, } \\
\text { MIMAT0003126 }\end{array}$ & $\begin{array}{l}\operatorname{miR}-133 a / b- \\
3 p\end{array}$ & -1.12 & 1.49 & 0.047 & * \\
\hline MIMAT0037263 & $\operatorname{miR}-16$ & -1.19 & 1.59 & 0.043 & * \\
\hline MIMAT0017131 & miR-145-3p & -1.29 & 1.83 & 0.049 & * \\
\hline MIMAT0017838 & miR-218b & -1.33 & 1.10 & 0.023 & * \\
\hline MIMAT0017851 & $\operatorname{miR}-3571$ & -1.35 & 1.43 & 0.014 & * \\
\hline MIMAT0017823 & miR-3596d & -1.39 & 1.08 & 0.042 & * \\
\hline MIMAT0017886 & miR-3596a & -1.45 & 1.11 & 0.041 & * \\
\hline MIMAT0017887 & miR-3588 & -1.58 & 1.25 & 0.030 & * \\
\hline MIMAT0000850 & $\operatorname{miR}-144-3 p$ & -2.30 & -1.16 & 0.037 & * \\
\hline$R S x \operatorname{sex}$ & & RS Male: Control Male & RS Female: Control Female & & \\
\hline MIMAT0000886 & $\operatorname{miR}-216 a-5 p$ & 1.48 & -1.35 & 0.035 & * \\
\hline MIMAT0017136 & miR-154-3p & 1.46 & -1.15 & 0.044 & * \\
\hline MIMAT0000801 & miR-29b-3p & 1.43 & -1.09 & 0.040 & * \\
\hline MIMAT0003195 & miR-376b-5p & 1.41 & -1.07 & 0.040 & * \\
\hline MIMAT0012833 & miR-582-5p & 1.39 & -1.27 & 0.025 & * \\
\hline MIMAT0004743 & miR-300-5p & 1.38 & -1.16 & 0.007 & * \\
\hline MIMAT0000552 & miR-301a-3p & 1.37 & -1.15 & 0.049 & * \\
\hline MIMAT0012834 & miR-592 & 1.35 & -1.18 & 0.033 & * \\
\hline MIMAT0000577 & miR-337-3p & 1.32 & -1.12 & 0.050 & * \\
\hline MIMAT0005301 & miR-188-5p & 1.30 & -1.11 & 0.032 & * \\
\hline MIMAT0004791 & miR-379-3p & 1.30 & -1.16 & 0.042 & * \\
\hline MIMAT0003196 & miR-376b-3p & 1.27 & -1.16 & 0.014 & * \\
\hline MIMAT0017143 & miR-186-3p & 1.24 & -1.14 & 0.031 & $*$ \\
\hline
\end{tabular}


Table 1 miRNAs altered by the interaction between maternal separation, restraint stress, and sex (Continued)

\begin{tabular}{|c|c|c|c|c|c|c|c|}
\hline & & CPM-Fold C & & & & & \\
\hline MIMAT0024845 & miR-3068-5p & 1.23 & & -1.18 & & 0.022 & * \\
\hline MIMAT0017223 & miR-374-3p & 1.20 & & -1.22 & & 0.046 & * \\
\hline MIMAT0017146 & miR-191a-3p & 1.19 & & -1.11 & & 0.027 & * \\
\hline MIMAT0000813 & $\operatorname{miR}-34 b-5 p$ & 1.18 & & -1.46 & & 0.016 & * \\
\hline MIMAT0017111 & miR-98-3p & 1.17 & & -1.17 & & 0.038 & * \\
\hline MIMAT0000888 & miR-218a-5p & 1.15 & & -1.22 & & 0.017 & * \\
\hline MIMAT0017213 & miR-541-3p & 1.14 & & -1.24 & & 0.007 & $*$ \\
\hline MIMAT0017127 & miR-138-2-3p & 1.14 & & -1.12 & & 0.018 & * \\
\hline MIMAT0017100 & $\operatorname{miR}-26 a-3 p$ & 1.13 & & -1.08 & & 0.024 & * \\
\hline MIMAT0005335 & miR-758-3p & 1.11 & & -1.11 & & 0.041 & * \\
\hline MIMAT0003191 & miR-493-3p & 1.09 & & -1.17 & & 0.022 & * \\
\hline MIMAT0017212 & miR-539-3p & 1.08 & & -1.30 & & 0.034 & * \\
\hline MIMAT0024846 & miR-3068-3p & 1.07 & & -1.13 & & 0.012 & * \\
\hline MIMAT0017874 & miR-3583-3p & 1.07 & & -1.17 & & 0.048 & * \\
\hline MIMAT0017307 & $\operatorname{miR}-434-5 p$ & 1.03 & & -1.26 & & 0.011 & * \\
\hline MIMAT0000791 & $\operatorname{miR}-22-3 p$ & 1.02 & & 1.41 & & 0.028 & * \\
\hline MIMAT0004733 & miR-136-3p & 1.02 & & -1.52 & & 0.048 & * \\
\hline MIMAT0005339 & miR-873-5p & 1.00 & & -1.24 & & 0.037 & * \\
\hline MIMAT0017137 & miR-181c-3p & -1.00 & & -1.26 & & 0.041 & $*$ \\
\hline MIMAT0017360 & miR-582-3p & -1.02 & & -1.46 & & 0.033 & * \\
\hline MIMAT0017117 & $\operatorname{miR}-127-5 p$ & -1.03 & & -1.27 & & 0.036 & $*$ \\
\hline MIMAT0001534 & miR-448-3p & -1.03 & & -1.54 & & 0.047 & * \\
\hline MIMAT0005331 & miR-708-5p & -1.04 & & 1.17 & & 0.033 & * \\
\hline MIMAT0005308 & $\operatorname{miR}-380-5 p$ & -1.06 & & -1.41 & & 0.041 & $*$ \\
\hline MIMAT0004705 & let-7b-3p & -1.06 & & 1.27 & & 0.041 & * \\
\hline MIMAT0004648 & miR-339-3p & -1.15 & & 1.18 & & 0.043 & $*$ \\
\hline MIMAT0017871 & miR-3596b & -1.19 & & 1.48 & & 0.012 & * \\
\hline MIMAT0017886 & miR-3596a & -1.20 & & 1.37 & & 0.031 & $*$ \\
\hline MIMAT0017877 & miR-3596c & -1.25 & & 1.36 & & 0.024 & $*$ \\
\hline MIMAT0000903 & $\operatorname{miR}-320-3 p$ & -1.28 & & 1.47 & & 0.037 & $*$ \\
\hline MIMAT0017094 & miR-16-3p & -1.28 & & 1.18 & & 0.017 & * \\
\hline MIMAT0005313 & miR-423-3p & -1.30 & & 1.52 & & 0.039 & * \\
\hline MIMAT0017866 & $\operatorname{miR}-3578$ & -1.33 & & 1.35 & & 0.031 & $*$ \\
\hline$M S \times R S \times \operatorname{sex}$ & & $\begin{array}{l}\text { MS Male: C } \\
\text { Male }\end{array}$ & $\begin{array}{l}\text { MS Female: C } \\
\text { Female }\end{array}$ & $\begin{array}{l}\text { MS RS Male: RS } \\
\text { Male }\end{array}$ & $\begin{array}{l}\text { MS RS Female: RS } \\
\text { Female }\end{array}$ & & \\
\hline MIMAT0017100 & $\operatorname{miR}-26 a-3 p$ & 1.21 & -1.02 & -1.07 & 1.25 & 0.009 & $*$ \\
\hline
\end{tabular}

All miRNAs with a significant main effect or interaction effect are listed here. CPM-fold change is presented as a ratio one group's miRNA expression over another group (usually the comparison control). Positive values indicate upregulation and negative values show downregulation. ${ }^{*} p<0.05,{ }^{* *} p<0.005,{ }^{* * *} p<0.001$.

Abbreviations: CPM-counts per million, MS-maternal separation, RS-restraint stress, C-control

and MS $(\mathrm{p}<0.001)$ animals $(\mathrm{F}(2,69)=17.077, p<0.001)$. One-way ANOVA revealed significant differences $(\mathrm{F}(2,69)=3.445, p<0.05)$ in EPM closed arm time (Fig. $1 \mathrm{x})$, but follow-up pairwise t-tests only showed a nonsignificant reduction in MS $(p=0.077)$ and MS + Enr $(p=$ $0.08)$ groups compared to controls. Enrichment significantly reduced adrenal weight (Fig. 1y) compared to both control $(p<0.005)$ and MS $(p<0.05)$ groups $(\mathrm{F}(2,69)=6.275, p<0.005)$. Plasma CORT levels showed no significant group differences $(\mathrm{F}(2,69)=0.485, p>0.05)$.

miRNA expression changes after MS and MS + Enrichment A one-way ANOVA comparing control, MS, and MS + Enr miRNA counts per million (CPM) expression values 
Table 2 miRNAs altered by the interaction between restraint stress, environmental enrichment, and sex in maternally separated rats

\begin{tabular}{|c|c|c|c|c|}
\hline & & CPM-Fold Change & & \\
\hline Accession ID & miRNA & & $p$ & Sig. \\
\hline restraint stress & & RS:Control & & \\
\hline MIMAT0004646 & miR-338-5p & 1.20 & 0.018 & $*$ \\
\hline MIMAT0000587 & miR-341 & -1.13 & 0.043 & $*$ \\
\hline enrichment & & Enr:Non-Enr & & \\
\hline MIMAT0017895 & miR-3592 & 1.37 & 0.002 & $* *$ \\
\hline MIMAT0017094 & $\operatorname{miR}-16-3 p$ & 1.30 & 0.002 & $* *$ \\
\hline MIMAT0017130 & $\operatorname{miR}-144-5 p$ & 1.28 & 0.036 & $*$ \\
\hline MIMAT0017158 & $\operatorname{miR}-212-5 p$ & 1.28 & 0.022 & $*$ \\
\hline MIMAT0017818 & miR-3556b & 1.27 & 0.001 & $* * *$ \\
\hline MIMAT0017836 & $\operatorname{miR}-9 b-3 p$ & 1.26 & 0.022 & * \\
\hline MIMAT0005299 & $\begin{array}{l}\text { miR-181d- } \\
5 p\end{array}$ & 1.26 & 0.002 & $* *$ \\
\hline MIMAT0000857 & $\begin{array}{l}\text { miR-181c- } \\
5 p\end{array}$ & 1.25 & 0.007 & * \\
\hline MIMAT0017357 & miR-362-3p & 1.24 & 0.007 & * \\
\hline MIMAT0000820 & miR-99a-5p & 1.23 & 0.024 & $*$ \\
\hline MIMAT0017850 & $\operatorname{miR}-3570$ & 1.22 & 0.015 & * \\
\hline MIMAT0017823 & miR-3596d & 1.22 & 0.010 & * \\
\hline MIMAT0005341 & $m i R-488-3 p$ & 1.20 & 0.021 & $*$ \\
\hline MIMAT0017360 & $\operatorname{miR}-582-3 p$ & 1.20 & 0.029 & $*$ \\
\hline MIMAT0017867 & $\operatorname{miR}-3579$ & 1.18 & 0.028 & * \\
\hline MIMAT0003207 & $\operatorname{miR}-369-3 p$ & 1.17 & 0.005 & $*$ \\
\hline MIMAT0005282 & $\operatorname{miR}-872-5 p$ & 1.14 & 0.003 & $* *$ \\
\hline MIMAT0000867 & miR-192-5p & 1.14 & 0.027 & $*$ \\
\hline MIMAT0004710 & $\begin{array}{l}\operatorname{miR}-17-1- \\
3 p\end{array}$ & 1.13 & 0.031 & $*$ \\
\hline MIMAT0017031 & miR-329-5p & 1.13 & 0.009 & $*$ \\
\hline MIMAT0003123 & miR-377-3p & 1.13 & 0.009 & $*$ \\
\hline MIMAT0003204 & $\begin{array}{l}\text { miR-409a- } \\
5 p\end{array}$ & 1.12 & 0.047 & $*$ \\
\hline MIMAT0000553 & miR-324-5p & -1.09 & 0.049 & * \\
\hline MIMAT0017371 & miR-666-3p & -1.13 & 0.012 & $*$ \\
\hline MIMAT0000794 & miR-24-3p & -1.14 & 0.022 & $*$ \\
\hline MIMAT0000562 & let-7d-5p & -1.16 & 0.022 & $*$ \\
\hline MIMAT0000784 & miR-15b-5p & -1.16 & 0.020 & * \\
\hline MIMAT0035726 & miR-149-5p & -1.17 & 0.029 & $*$ \\
\hline MIMAT0004729 & $\begin{array}{l}\operatorname{miR}-125 a- \\
3 p\end{array}$ & -1.18 & 0.035 & $*$ \\
\hline MIMAT0017874 & $\begin{array}{l}\text { miR-3583- } \\
3 p\end{array}$ & -1.21 & 0.000 & $* * *$ \\
\hline MIMAT0012845 & miR-935 & -1.21 & 0.028 & $*$ \\
\hline MIMAT0017318 & miR-873-3p & -1.22 & 0.031 & * \\
\hline MIMAT0000570 & miR-331-3p & -1.23 & 0.022 & $*$ \\
\hline MIMAT0000853 & miR-150-5p & -1.26 & 0.033 & * \\
\hline MIMAT0001549 & miR-365-3p & -1.27 & 0.004 & $* *$ \\
\hline
\end{tabular}


Table 2 miRNAs altered by the interaction between restraint stress, environmental enrichment, and sex in maternally separated rats (Continued)

\begin{tabular}{|c|c|c|c|c|c|}
\hline & & CPM-Fold Change & & & \\
\hline MIMAT0001543 & $\begin{array}{l}\text { miR-449a- } \\
5 p\end{array}$ & -1.33 & & 0.035 & $*$ \\
\hline MIMAT0004742 & miR-296-3p & -1.36 & & 0.027 & $*$ \\
\hline MIMAT0035752 & miR-762 & -1.37 & & 0.029 & * \\
\hline $\begin{array}{l}\text { MIMAT00000839, } \\
\text { MIMAT0003126 }\end{array}$ & $\begin{array}{l}\operatorname{miR}-133 a l \\
b-3 p\end{array}$ & -1.42 & & 0.026 & * \\
\hline sex & & Female:Male & & & \\
\hline MIMAT0035719 & let-7g-5p & 1.15 & & 0.030 & * \\
\hline MIMAT0000819 & miR-98-5p & 1.14 & & 0.008 & * \\
\hline MIMAT0003154 & $\operatorname{miR}-29 c-5 p$ & 1.13 & & 0.033 & * \\
\hline MIMAT0017175 & $\operatorname{miR}-421-3 p$ & 1.12 & & 0.002 & $* *$ \\
\hline MIMAT0005309 & $\operatorname{miR}-384-5 p$ & 1.11 & & 0.022 & $*$ \\
\hline MIMAT0000796 & $\operatorname{miR}-26 a-5 p$ & 1.09 & & 0.012 & * \\
\hline MIMAT0024846 & $\begin{array}{l}\operatorname{miR}-3068- \\
3 p\end{array}$ & -1.07 & & 0.023 & * \\
\hline MIMAT0005342 & $\operatorname{miR}-652-3 p$ & -1.10 & & 0.015 & * \\
\hline MIMAT0005278 & $\begin{array}{l}\text { miR-466b- } \\
5 p\end{array}$ & -1.10 & & 0.035 & $*$ \\
\hline MIMAT0005311 & miR-410-3p & -1.16 & & 0.050 & * \\
\hline MIMAT0004715 & $\operatorname{miR}-27 a-5 p$ & -1.18 & & 0.027 & * \\
\hline MIMAT0000887 & miR-217-5p & -1.23 & & 0.039 & * \\
\hline MIMAT0025049 & $\operatorname{miR}-344 i$ & -1.25 & & 0.008 & $*$ \\
\hline MIMAT0012851 & $\operatorname{miR}-547-3 p$ & -2.51 & & 0.000 & *** \\
\hline$R S \times E n r$ & & Enr:Non-Enr & Enr + RS:Non-Enr + RS & & \\
\hline MIMAT0017043 & $\begin{array}{l}\text { miR-135b- } \\
3 p\end{array}$ & -1.04 & 1.27 & 0.022 & $*$ \\
\hline$R S x \operatorname{sex}$ & & RS Male:Control Male & RS Female:Control Female & & \\
\hline MIMAT0017371 & $\operatorname{miR}-666-3 p$ & 1.12 & -1.09 & 0.043 & * \\
\hline Enr $x$ sex & & Enr Male:Non-Enr Male & Enr Female:Non-Enr Female & & \\
\hline MIMAT0017887 & $\operatorname{miR}-3588$ & 1.45 & -1.11 & 0.035 & $*$ \\
\hline MIMAT0004705 & let-7b-3p & 1.23 & -1.09 & 0.014 & * \\
\hline MIMAT0017877 & miR-3596c & 1.23 & -1.12 & 0.049 & * \\
\hline MIMAT0000574 & $\operatorname{miR}-140-3 p$ & 1.22 & -1.18 & 0.036 & * \\
\hline MIMAT0000583 & $\operatorname{miR}-339-5 p$ & 1.20 & -1.17 & 0.016 & * \\
\hline MIMAT0003378 & $\begin{array}{l}\text { miR-378a- } \\
5 p\end{array}$ & 1.16 & -1.36 & 0.009 & * \\
\hline MIMAT0003213 & $\operatorname{miR}-503-5 p$ & 1.15 & -1.47 & 0.033 & * \\
\hline MIMAT0017109 & $\operatorname{miR}-93-3 p$ & 1.13 & -1.20 & 0.048 & * \\
\hline MIMAT0000830 & $\begin{array}{l}\text { miR-125b- } \\
5 p\end{array}$ & 1.11 & -1.21 & 0.028 & * \\
\hline MIMAT0004648 & miR-339-3p & 1.10 & -1.27 & 0.036 & $*$ \\
\hline MIMAT0000608 & $\operatorname{miR}-351-5 p$ & 1.10 & -1.71 & 0.049 & $*$ \\
\hline MIMAT0005299 & $\begin{array}{l}\text { miR-181d- } \\
5 p\end{array}$ & 1.09 & 1.46 & 0.047 & * \\
\hline MIMAT0000792 & miR-23a-3p & 1.08 & -1.24 & 0.045 & * \\
\hline MIMAT0004739 & miR-204-3p & 1.07 & -1.75 & 0.034 & * \\
\hline
\end{tabular}


Table 2 miRNAs altered by the interaction between restraint stress, environmental enrichment, and sex in maternally separated rats (Continued)

\begin{tabular}{|c|c|c|c|c|c|}
\hline & & $C P M-F$ & & & \\
\hline MIMAT0017224 & miR-503-3p & 1.07 & -1.54 & 0.048 & * \\
\hline MIMAT0025067 & miR-6328 & 1.06 & -1.34 & 0.043 & * \\
\hline MIMAT0005282 & $\operatorname{miR}-872-5 p$ & 1.04 & 1.26 & 0.030 & * \\
\hline MIMAT0000774 & let-7a-5p & 1.01 & -1.16 & 0.031 & * \\
\hline MIMAT0001549 & $\operatorname{miR}-365-3 p$ & -1.07 & -1.48 & 0.038 & $*$ \\
\hline MIMAT0005335 & miR-758-3p & -1.07 & 1.22 & 0.017 & * \\
\hline MIMAT0005311 & $\operatorname{miR}-410-3 p$ & -1.08 & 1.36 & 0.016 & * \\
\hline MIMAT0025070 & miR-6331 & -1.11 & 1.36 & 0.012 & * \\
\hline MIMAT0004718 & $\operatorname{miR}-29 a-5 p$ & -1.11 & 1.28 & 0.038 & * \\
\hline MIMAT0005287 & miR-879-5p & -1.14 & 1.28 & 0.048 & $*$ \\
\hline MIMAT0017136 & miR-154-3p & -1.15 & 1.70 & 0.024 & $*$ \\
\hline MIMAT0017102 & $\operatorname{miR}-31 a-3 p$ & -1.21 & 1.32 & 0.040 & * \\
\hline MIMAT0001628 & miR-433-3p & -1.22 & 1.24 & 0.003 & $* *$ \\
\hline MIMAT0017111 & miR-98-3p & -1.23 & 1.13 & 0.030 & * \\
\hline MIMAT0000568 & $\operatorname{miR}-330-3 p$ & -1.23 & 1.15 & 0.046 & $*$ \\
\hline MIMAT0003381 & miR-499-5p & -1.24 & 1.18 & 0.042 & $*$ \\
\hline MIMAT0000611 & $\begin{array}{l}\text { miR-135b- } \\
5 p\end{array}$ & -1.25 & 1.30 & 0.020 & * \\
\hline MIMAT0005279 & $\begin{array}{l}\operatorname{miR}-466 c^{-} \\
5 p\end{array}$ & -1.25 & 1.41 & 0.020 & * \\
\hline MIMAT0004791 & miR-379-3p & -1.27 & 1.32 & 0.034 & * \\
\hline MIMAT0000813 & $\operatorname{miR}-34 b-5 p$ & -1.27 & 1.38 & 0.014 & * \\
\hline MIMAT0000557 & miR-325-5p & -1.28 & 1.35 & 0.017 & * \\
\hline MIMAT0017211 & $\operatorname{miR}-540-5 p$ & -1.29 & 1.28 & 0.011 & * \\
\hline MIMAT0000613 & miR-151-5p & -1.30 & 1.25 & 0.043 & * \\
\hline MIMAT0000811 & miR-32-5p & -1.31 & 1.28 & 0.038 & $*$ \\
\hline MIMAT0000865 & $\begin{array}{l}\text { miR-190a- } \\
5 p\end{array}$ & -1.31 & 1.20 & 0.026 & * \\
\hline MIMAT0017104 & miR-33-3p & -1.32 & 1.16 & 0.041 & $*$ \\
\hline MIMAT0012833 & $\operatorname{miR}-582-5 p$ & -1.32 & 1.47 & 0.038 & $*$ \\
\hline MIMAT0012834 & miR-592 & -1.33 & 1.38 & 0.022 & * \\
\hline MIMAT0000577 & miR-337-3p & -1.33 & 1.29 & 0.041 & * \\
\hline MIMAT0017302 & miR-380-3p & -1.34 & 1.32 & 0.034 & * \\
\hline MIMAT0024845 & $\begin{array}{l}\operatorname{miR}-3068- \\
5 p\end{array}$ & -1.34 & 1.12 & 0.037 & * \\
\hline MIMAT0000787 & miR-18a-5p & -1.35 & 1.28 & 0.023 & * \\
\hline MIMAT0017192 & $\operatorname{miR}-433-5 p$ & -1.39 & 1.27 & 0.011 & * \\
\hline MIMAT0000789 & $\operatorname{miR}-19 a-3 p$ & -1.42 & 1.36 & 0.044 & * \\
\hline MIMAT0003195 & $\begin{array}{l}\text { miR-376b- } \\
5 p\end{array}$ & -1.43 & 1.21 & 0.048 & * \\
\hline MIMAT0000552 & $\begin{array}{l}\text { miR-301a- } \\
3 p\end{array}$ & -1.44 & 1.30 & 0.028 & * \\
\hline MIMAT0000825 & $\begin{array}{l}\text { miR-106b- } \\
5 p\end{array}$ & -1.45 & 1.23 & 0.049 & * \\
\hline MIMAT0000801 & miR-29b-3p & -1.46 & 1.35 & 0.039 & * \\
\hline MIMAT0000855 & miR-153-3p & -1.49 & 1.39 & 0.027 & * \\
\hline
\end{tabular}


Table 2 miRNAs altered by the interaction between restraint stress, environmental enrichment, and sex in maternally separated rats (Continued)

\begin{tabular}{|c|c|c|c|c|c|c|c|}
\hline & & CPM-Fold Change & & & & & \\
\hline MIMAT0000842 & miR-136-5p & -1.51 & & 1.41 & & 0.021 & $*$ \\
\hline MIMAT0017135 & $\operatorname{miR}-153-5 p$ & -1.55 & & 1.35 & & 0.022 & * \\
\hline MIMAT0003176 & miR-539-5p & -1.59 & & 1.84 & & 0.020 & $*$ \\
\hline$R S \times$ Enr $x$ sex & & $\begin{array}{l}\text { Enr Male: Non-Enr } \\
\text { Male }\end{array}$ & $\begin{array}{l}\text { Enr Female: Non-Enr } \\
\text { Female }\end{array}$ & $\begin{array}{l}\text { Enr + RS Male: Non-Enr } \\
\text { RS Male }\end{array}$ & $\begin{array}{l}\text { Enr RS Female: Non-Enr RS } \\
\text { Female }\end{array}$ & & \\
\hline MIMAT0005322 & $\operatorname{miR}-532-5 p$ & 1.32 & -1.03 & -1.04 & 1.24 & 0.041 & * \\
\hline MIMAT0017090 & let-7f-2-3p & 1.27 & 1.02 & -1.28 & 1.67 & 0.024 & * \\
\hline MIMAT0000827 & miR-122-5p & 1.21 & -1.39 & -1.04 & 1.17 & 0.038 & $*$ \\
\hline MIMAT0017139 & $\begin{array}{l}\text { miR-181b- } \\
1-3 p\end{array}$ & 1.20 & -1.18 & -1.13 & 1.25 & 0.013 & * \\
\hline MIMAT0035748 & $\operatorname{miR}-452-5 p$ & 1.18 & -1.30 & -1.11 & 1.40 & 0.024 & * \\
\hline MIMAT0000803 & $\operatorname{miR}-29 c-3 p$ & 1.17 & 1.01 & -1.14 & 1.37 & 0.048 & * \\
\hline MIMAT0004705 & let-7b-3p & 1.16 & 1.13 & 1.31 & -1.33 & 0.025 & * \\
\hline MIMAT0000615 & $\begin{array}{l}\text { miR-101b- } \\
3 p\end{array}$ & 1.12 & -1.09 & -1.15 & 1.31 & 0.040 & * \\
\hline MIMAT0012838 & $\operatorname{miR}-653-5 p$ & 1.12 & -1.11 & -1.12 & 1.49 & 0.026 & * \\
\hline MIMAT0012831 & $\operatorname{miR}-544-3 p$ & 1.12 & -1.12 & -1.34 & 1.47 & 0.024 & * \\
\hline MIMAT0017143 & miR-186-3p & 1.09 & -1.08 & -1.47 & 1.26 & 0.027 & * \\
\hline MIMAT0024846 & $\begin{array}{l}\operatorname{miR}-3068- \\
3 p\end{array}$ & 1.09 & -1.03 & -1.08 & 1.12 & 0.010 & $*$ \\
\hline MIMAT0005282 & miR-872-5p & 1.08 & 1.10 & -1.00 & 1.44 & 0.041 & $*$ \\
\hline MIMAT0004711 & $\operatorname{miR}-21-3 p$ & 1.06 & -1.41 & -1.38 & 1.24 & 0.026 & * \\
\hline MIMAT0017877 & miR-3596c & 1.06 & 1.12 & 1.43 & -1.40 & 0.020 & * \\
\hline MIMAT0017033 & miR-331-5p & 1.05 & -1.10 & -1.04 & 1.18 & 0.013 & * \\
\hline MIMAT0017871 & miR-3596b & -1.01 & 1.15 & 1.34 & -1.42 & 0.010 & * \\
\hline MIMAT0000791 & $\operatorname{miR}-22-3 p$ & -1.04 & 1.11 & 1.15 & -1.35 & 0.023 & * \\
\hline MIMAT0000774 & let-7a-5p & -1.07 & -1.06 & 1.10 & -1.26 & 0.029 & * \\
\hline MIMAT0000862 & miR-185-5p & -1.12 & -1.01 & 1.19 & -1.31 & 0.019 & * \\
\hline MIMAT0000560 & miR-326-3p & -1.14 & 1.04 & 1.13 & -1.32 & 0.037 & * \\
\hline MIMAT0000810 & $\operatorname{miR}-31 a-5 p$ & -1.16 & 1.05 & 1.26 & -1.28 & 0.036 & * \\
\hline MIMAT0017802 & miR-3547 & -1.18 & -1.01 & 1.31 & -1.59 & 0.027 & * \\
\hline MIMAT0000562 & let-7d-5p & -1.23 & -1.08 & 1.08 & -1.44 & 0.027 & * \\
\hline MIMAT0025067 & miR-6328 & -1.23 & -1.19 & 1.44 & -1.50 & 0.027 & $*$ \\
\hline MIMAT0035726 & miR-149-5p & -1.23 & -1.09 & 1.07 & -1.43 & 0.045 & * \\
\hline MIMAT0000554 & miR-324-3p & -1.24 & -1.09 & 1.33 & -1.52 & 0.023 & * \\
\hline MIMAT0004641 & miR-330-5p & -1.24 & -1.00 & 1.07 & -1.20 & 0.022 & * \\
\hline MIMAT0012844 & $\operatorname{miR}-665$ & -1.30 & -1.06 & 1.11 & -1.36 & 0.039 & * \\
\hline MIMAT0012845 & miR-935 & -1.33 & -1.18 & 1.18 & -1.59 & 0.035 & * \\
\hline MIMAT0000853 & miR-150-5p & -1.48 & -1.08 & 1.15 & -1.71 & 0.018 & * \\
\hline MIMAT0017885 & miR-702-3p & -1.54 & -1.11 & 1.24 & -1.68 & 0.039 & * \\
\hline
\end{tabular}

All animal subjects in this comparison received MS-180. miRNAs with a significant main effect or interaction effect are listed here. CPM-fold change is presented as a ratio one group's miRNA expression over another group (usually the comparison control). Positive values indicate upregulation and negative values show downregulation. ${ }^{*} p<0.05,{ }^{* *} p<0.005,{ }^{* * *} p<0.001$. Abbreviations: CPM-counts per million, MS-maternal separation, RS-restraint stress, Enr-environmental enrichment, C-control 
showed that 29 miRNAs were significantly upregulated by MS and 21 were significantly downregulated. These changes include miRNAs which were only affected by MS as well as those only affected by MS + Enr or by both MS and MS + Enr. CPM-fold change and $p$ values are shown in Table 3 and describe specific expression changes across each of the three groups. miRs-144-3p and - 206-5p exhibited the greatest fold change at 1.783and 1.766-fold downregulation in MS animals compared to controls $(p<0.05)$. miR-29b-1-5p, $-301 \mathrm{~b}-3 \mathrm{p}$, and 3065-5p showed a treatment response to Enr in that MS significantly downregulated their expression $(p<0.05)$ and Enr, at least partially, reversed this effect. Changes specific to male and female animals are shown in Supplementary Tables 1 and 2 .

We visualized the chromosomal location of each significantly altered miRNA in Fig. 2a. 12 miRNAs grouped together on chromosome 6 and each of these were upregulated by MS except for miRs-673-5p and - 341. 7 miRNAs grouped on chromosome 10.

\section{miRNA gene targets}

We used Ingenuity Pathway Analysis (IPA; Hilden, Germany) to determine which miRNAs associated with MS and Enr shared common stress and depressionrelated gene targets and had the greatest number of these targets. Thus, miRNA-gene target maps were created based on significantly altered miRNAs and their gene targets. To determine hub miRNAs (i.e. those with the most targets and the most shared common targets), we filtered out miRNAs with 10 or fewer gene connections. 3418 gene targets were identified. miRs-132-5p, - 132-3p, $-449 a-5 p, 30 e-5 p,-338-3 p,-301 b-3 p,-144-3 p$, and let$7 \mathrm{~g}$-3p were hub miRNAs forming a highly integrated network with many shared gene targets (Fig. 2b). Gene ontology (GO) analysis (Fig. 2c) confirmed the involvement of these miRNA gene targets in MAPK signaling as well as Ras signaling, ion binding, and neuron part ontologies. The top ontologies for $\mathrm{GO}$ analysis are further described in Supplementary File 1. Hierarchical clustering of the GO terms in Metascape revealed that these pathways and functions were highly overlapped (Fig. 2d). Stress-related gene targets were selected for expression based on a literature search for anxiety, depression, stress, and related molecular pathways (Supplementary Table 3); we also included those genes with the greatest number of targeting miRNAs. 2-way ANOVA revealed that only GRIN2B expression was significantly altered $(\mathrm{F}(2,72)=3.703, p=$ 0.030 ) by the interaction between sex and group (control, MS, and MS + Enr) with only females showing increased expression following Enr, though not in controls or MS animals (Fig. 2e). Using pairwise t-tests, we found significant upregulation of GABRA1 $(\mathrm{t}(46)=2.035, p=0.048)$, MAPK6 $(\mathrm{t}(46)=2.307, p=0.026)$, and MMP19 $(\mathrm{t}(46)=$
2.156, $p=0.036)$ in MS $(n=24)$ animals compared to controls $(\mathrm{n}=24)$ (Fig. 2e). Several genes were upregulated in MS + Enr ( $\mathrm{n}=24)$ animals compared to controls $(\mathrm{n}=24)$, including CALM1 $(\mathrm{t}(46)=4.307, p<0.000)$, ESR1 $(\mathrm{t}(46)=$ $2.520, \quad p=0.015), \quad$ FOXO1 $\quad(\mathrm{t}(46)=2.137, \quad p=0.038)$, GABRA1 $(\mathrm{t}(46)=2.384, p=0.021)$, IL10RB $(\mathrm{t}(46)=2.271$, $p=0.028), \quad$ KALRN $\quad(\mathrm{t}(46)=3.634, \quad p=0.001), \quad$ PIK3CB $(\mathrm{t}(46)=3.077, p=0.004), \quad$ TBP $\quad(\mathrm{t}(46)=2.307, p=0.026)$, TRAF6 $(\mathrm{t}(46)=2.320, p=0.025)$, TSC22D3 $(\mathrm{t}(46)=2.313$, $p=0.025)$, and WNT2B $(\mathrm{t}(46)=2.697, p=0.010)$. Only two genes showed expression changes following MS that returned to the level of controls following Enr: MAPK6 and MMP19.

\section{Exploring methylation as a miRNA regulatory mechanism}

Of the 50 miRNAs significantly altered by MS, we found 5 miRNAs (miRs-219a, - 207, - 132, - 212, and let-7i) with CPG islands within $1000 \mathrm{~kb}$ of their promoter region. miRs-132 and -212 localized closely on chromosome 10 and shared a CPG island in their promoter regions (Fig. 2f). Pairwise t-test revealed increased methylation at the promoter region of miR-219a in MS (2.61-fold increase, $\mathrm{t}(30)=2.29, p=0.029 ; n=4$ male and $\mathrm{n}=4$ female per group) but not MS + Enr animals (Fig. $2 \mathrm{~g})$. There were no significant group differences in methylation for let-7i, miR-207, or miR-132/212 ( $p>$ 0.05).

\section{Discussion}

In this study, we present the first genome-wide profiling of miRNAs in the hypothalamus following MS and RS. We also parsed sex and estrus phase differences in miRNA expression resulting from these stress paradigms. Finally, we show miRNA changes underlying the use of environmental enrichment as a preventative for ELS-induced depressive-behavior. We found that 29 miRNAs were upregulated and 21 were downregulated by MS. Out of 21 downregulated miRNAs, 3 miRNAs (miR-29b-1-5p, -301b-3p, and-3065-5p) showed expression levels similar to controls in MS + Enr animals. Chromosomal localization revealed two large groups of MS-induced miRNAs on chromosome 6 and chromosome 10. Based on significantly altered miRNAs in MS, we detected over 3418 miRNA gene targets. qPCR-based expression analysis showed that GABRA1, MAPK6, and MMP19 were significantly increased in MS animals compared to controls. Interestingly, MAPK6 and MMP19 expression were reversed by Enr in MS animals. miRNA-gene target mapping revealed several miRNA regulatory hubs including miR-301b-3p, - 132-3p, 132-5p, -449a-5p, -30e-5p, - 338-3p, - 144-3p, and let$7 \mathrm{~g}-3 \mathrm{p}$. GO analysis confirmed that these miRNA gene targets were significantly involved in MAPK signaling. Finally, we found that a CPG island near the promoter 
Table $\mathbf{3}$ miRNAs significantly altered by MS and Enrichment

\begin{tabular}{|c|c|c|c|c|c|c|c|}
\hline \multirow[b]{2}{*}{ Accession ID } & \multirow[b]{2}{*}{ Chromosomal Location } & \multirow[b]{2}{*}{ miRNA } & \multicolumn{3}{|c|}{ CPM-Fold Change } & \multirow[t]{2}{*}{$p$ (one-way ANOVA) } & \multirow[b]{2}{*}{ sig. } \\
\hline & & & MS:C & MS + Enr:C & MS + Enr:MS & & \\
\hline \multicolumn{8}{|l|}{ upregulated by MS } \\
\hline MIMAT0017819 & chr18: 56726128-56,726,240 & $\operatorname{miR}-3557-5 p$ & 1.282 & 1.359 & 1.060 & 0.043 & $b, d$ \\
\hline MIMAT0000838 & chr10: 62014995-62,015,095 & $\operatorname{miR}-132-3 p$ & 1.278 & 1.394 & 1.091 & 0.000 & $a, b, d, f$ \\
\hline MIMAT0003379 & chr18: 56726150-56,726,214 & $\operatorname{miR}-378 a-3 p$ & 1.276 & 1.362 & 1.068 & 0.019 & $a, b, d$ \\
\hline MIMAT0026467 & chr11: 16097346-16,097,433 & $\operatorname{miR}-125 b-2-3 p$ & 1.252 & 1.425 & 1.138 & 0.034 & $b, d, f$ \\
\hline MIMAT0017158 & chr10: 62014702-62,014,812 & $\operatorname{miR}-212-5 p$ & 1.252 & 1.598 & 1.276 & 0.000 & $a, b, c, d, f$ \\
\hline MIMAT0035730 & chr13: 77065446-77,065,507 & $\operatorname{miR}-1843 b-5 p$ & 1.201 & 1.263 & 1.051 & 0.015 & $a, b, d$ \\
\hline MIMAT0000859 & chr13: 54952903-54,953,012 & miR-181b-5p & 1.195 & 1.373 & 1.149 & 0.004 & $a, b, d, f$ \\
\hline MIMAT0003205 & chr6: 133893419-133,893,495 & $\operatorname{miR}-409 a-3 p$ & 1.176 & 1.324 & 1.125 & 0.000 & $a, b, d, f$ \\
\hline MIMAT0000822 & chr8: 45746948-45,747,027 & $\operatorname{miR}-100-5 p$ & 1.176 & 1.436 & 1.221 & 0.002 & $b, d, f$ \\
\hline MIMAT0000550 & chr6: 133861199-133,861,284 & $\operatorname{miR}-323-3 p$ & 1.163 & 1.275 & 1.097 & 0.001 & $a, b, d, f$ \\
\hline MIMAT0000820 & chr11: 16052153-16,052,233 & $\operatorname{miR}-99 a-5 p$ & 1.161 & 1.431 & 1.232 & 0.000 & $b, c, d, f$ \\
\hline MIMAT0017163 & chrX: 3684480-3,684,588 & $\operatorname{miR}-221-5 p$ & 1.130 & 1.305 & 1.156 & 0.001 & $b, c, d, f$ \\
\hline MIMAT0017217 & chr6: 133699509-133,699,592 & miR-493-5p & 1.124 & 1.220 & 1.085 & 0.046 & $b, d, f$ \\
\hline MIMAT0003202 & chr6: 133884178-133,884,257 & $\operatorname{miR}-382-3 p$ & 1.123 & 1.310 & 1.167 & 0.002 & $b, c, d, f$ \\
\hline MIMAT0001626 & chr6: 133711425-133,711,538 & $\operatorname{miR}-431$ & 1.118 & 1.225 & 1.096 & 0.002 & $a, b, d, f$ \\
\hline MIMAT0000858 & chr3: 23150352-23,150,468 & $\operatorname{miR}-181 a-5 p$ & 1.117 & 1.343 & 1.203 & 0.021 & $b, d, f$ \\
\hline MIMAT0017123 & chr10: 62014995-62,015,095 & miR-132-5p & 1.115 & 1.249 & 1.120 & 0.002 & $a, b, d, f$ \\
\hline MIMAT0005299 & chr19: 25290051-25,290,133 & miR-181d-5p & 1.113 & 1.399 & 1.257 & 0.000 & $b, c, d, f$ \\
\hline MIMAT0003177 & chr6: 133892655-133,892,744 & $\operatorname{miR}-541-5 p$ & 1.108 & 1.265 & 1.142 & 0.012 & $b, d, f$ \\
\hline MIMAT0005314 & chr8: 117354821-117,354,903 & $\operatorname{miR}-425-5 p$ & 1.105 & 1.239 & 1.121 & 0.023 & $b, d, f$ \\
\hline MIMAT0003194 & chr6: 133872439-133,872,522 & $\operatorname{miR}-376 c-3 p$ & 1.103 & 1.185 & 1.074 & 0.002 & $a, b, d, f$ \\
\hline MIMAT0024843 & chr1: 143360224-143,360,287 & miR-1839-5p & 1.103 & 1.237 & 1.122 & 0.014 & $b, d, f$ \\
\hline MIMAT0017870 & chr6: 133893418-133,893,497 & $\operatorname{miR}-409 b$ & 1.101 & 1.173 & 1.065 & 0.045 & $b, d, f$ \\
\hline MIMAT0003204 & chr6: 133893419-133,893,495 & $\operatorname{miR}-409 a-5 p$ & 1.086 & 1.220 & 1.123 & 0.003 & $b, c, d, f$ \\
\hline MIMAT0004706 & chr1: 59704381-59,704,473 & let-7e-3p & 1.082 & 1.087 & 1.005 & 0.037 & $a, b, d$ \\
\hline MIMAT0004710 & chr15: 100179879-100,179,962 & $\operatorname{miR}-17-1-3 p$ & 1.069 & 1.212 & 1.134 & 0.001 & $b, c, d, f$ \\
\hline MIMAT0003200 & chr6: 133877124-133,877,205 & $\operatorname{miR}-487 b-3 p$ & 1.069 & 1.183 & 1.106 & 0.043 & $b, d, f$ \\
\hline MIMAT0003207 & chr6: 133893693-133,893,771 & $\operatorname{miR}-369-3 p$ & 1.061 & 1.237 & 1.166 & 0.000 & $b, c, d, f$ \\
\hline MIMAT0005282 & chr5: 113657727-113,657,807 & $\operatorname{miR}-872-5 p$ & 1.046 & 1.193 & 1.141 & 0.001 & $b, c, d, f$ \\
\hline \multicolumn{8}{|c|}{ downregulated by MS } \\
\hline MIMAT0000850 & chr10: 65291365-65,291,447 & miR-144-3p & -1.783 & -1.701 & 1.048 & 0.024 & $a, d$, \\
\hline MIMAT0017154 & chr9: 26791764-26,791,847 & miR-206-5p & -1.766 & -1.707 & 1.035 & 0.008 & $a, b, d$ \\
\hline MIMAT0005445 & chr4: 58344310-58,344,390 & miR-29b-1-5p & -1.708 & -1.520 & 1.124 & 0.012 & $a, b, d, e$, \\
\hline MIMAT0005304 & chr11: 88129426-88,129,503 & $\operatorname{miR}-301 b-3 p$ & -1.564 & -1.335 & 1.171 & 0.036 & $a, d, e$, \\
\hline MIMAT0017230 & chr10: 56844977-56,845,045 & miR-497-3p & -1.386 & -1.582 & -1.142 & 0.001 & $a, b, d, f$ \\
\hline MIMAT0000889 & chr20: 3816158-3,816,267 & $\operatorname{miR}-219 a-5 p$ & -1.314 & -1.504 & -1.144 & 0.010 & $b, d, f$ \\
\hline MIMAT0000812 & chr7: 123431612-123,431,680 & $\operatorname{miR}-33-5 p$ & -1.311 & -1.620 & -1.235 & 0.003 & $a, b, d, f$ \\
\hline MIMAT0001543 & chr2: 44897601-44,897,691 & $\operatorname{miR}-449 a-5 p$ & -1.251 & -1.660 & -1.327 & 0.001 & $b, c, d, f$ \\
\hline MIMAT0017839 & chr10: 109195234-109,195,336 & miR-3065-5p & -1.238 & -1.139 & 1.087 & 0.045 & $a, d$ \\
\hline MIMAT0035720 & chr8: 114874457-114,874,544 & let-7 $g-3 p$ & -1.231 & -1.288 & -1.046 & 0.017 & $a, b, d$, \\
\hline MIMAT0005328 & chr6: 133691381-133,691,463 & $\operatorname{miR}-673-5 p$ & -1.210 & -1.306 & -1.080 & 0.000 & $a, b, d, f$ \\
\hline MIMAT0017143 & chr2: 263873759-263,873,844 & $\operatorname{miR}-186-3 p$ & -1.205 & -1.253 & -1.040 & 0.015 & $a, b, d$, \\
\hline
\end{tabular}


Table 3 miRNAs significantly altered by MS and Enrichment (Continued)

\begin{tabular}{|c|c|c|c|c|c|c|c|}
\hline \multirow[b]{2}{*}{ Accession ID } & \multirow[b]{2}{*}{ Chromosomal Location } & \multirow[b]{2}{*}{ miRNA } & \multicolumn{3}{|c|}{ CPM-Fold Change } & \multirow[t]{2}{*}{$p$ (one-way ANOVA) } & \multirow[b]{2}{*}{ sig. } \\
\hline & & & MS:C & MS + Enr:C & MS + Enr:MS & & \\
\hline MIMAT0017026 & chr10: 74417746-74,417,845 & $\operatorname{miR}-301 a-5 p$ & -1.203 & -1.341 & -1.115 & 0.008 & $a, b, d, f$ \\
\hline MIMAT0000805 & chr5: 139702872-139,702,963 & $\operatorname{miR}-30 e-5 p$ & -1.178 & -1.238 & -1.052 & 0.007 & $a, b, d, f$ \\
\hline MIMAT0004707 & chr7: $66802731-66,802,815$ & let-7i-3p & -1.168 & -1.326 & -1.135 & 0.006 & $b, d, f$ \\
\hline MIMAT0000581 & chr10: 109195251-109,195,316 & $\operatorname{miR}-338-3 p$ & -1.147 & -1.277 & -1.113 & 0.024 & $b, d, f$ \\
\hline MIMAT0017093 & chr2: 165605923-165,606,020 & $\operatorname{miR}-15 b-3 p$ & -1.144 & -1.174 & -1.027 & 0.042 & $b, d$, \\
\hline MIMAT0000587 & chr6: 133733240-133,733,335 & $\operatorname{miR}-341$ & -1.131 & -1.196 & -1.057 & 0.020 & $b, d, f$ \\
\hline MIMAT0004732 & chr7: 32894877-32,894,976 & $\operatorname{miR}-135 a-3 p$ & -1.114 & -1.189 & -1.068 & 0.007 & $b, d, f$ \\
\hline MIMAT0000815 & chr5: 167092491-167,092,592 & $\operatorname{miR}-34 a-5 p$ & -1.090 & -1.241 & -1.139 & 0.036 & $b, d, f$ \\
\hline MIMAT0000570 & chr7: 34881095-34,881,190 & $\operatorname{miR}-331-3 p$ & -1.067 & -1.315 & -1.233 & 0.001 & $b, c, d, f$ \\
\hline \multicolumn{8}{|c|}{$\begin{array}{l}\text { a controls vs. MS } \\
\text { b controls vs. MS + Enr } \\
\text { c MS vs. MS + Enr } \\
\text { d controls vs. MS and MS + Enr } \\
\text { e controls and MS + Enr vs. MS (recovered by Enr) } \\
\text { f controls and MS vs. MS + Enr } \\
\text { Significant changes in miRNA expression after MS and Enr based on one-way ANOVA are shown. Listed miRNAs are split by those upregulated vs. downregulated } \\
\text { by MS. CPM-fold change value was calculated as a ratio between two group's CPM expression. Positive values indicate increased expression and negative values } \\
\text { show reduced expression in the numerator group. The full one-way ANOVA significance is listed as } p \text {. Specific group differences are listed as sig. Abbreviations: } \\
\text { CPM-counts per million, MS-maternal separation, Enr-environmental enrichment, C-control }\end{array}$} \\
\hline
\end{tabular}

of miR-219 was hypermethylated in MS but not significantly reversed by Enr.

MDD onset is usually preceded by a recent stressful event [3]. We did not find that RS precipitated a depression-like phenotype in MS animals. In female MS animals, RS increased escape latency but still this did not reach levels associated with learned helplessness (> $20 \mathrm{~s}$ latency [42];). Alternatively, each group variable (MS and RS) was associated with unique behavioral profiles. MS significantly decreased sucrose preference indicating increased anhedonia whereas RS decreased total movement in the EPM. However, RS-only males spent increased time in the closed arms of the EPM which has been mostly associated with increased anxiety [43]. RS animals also exhibited increased CORT levels compared to controls and MS without RS. Studies on ELS often report altered stress reactivity meaning that CORT may be more elevated following an acute stressor in individuals who experience ELS compared to those who have no ELS history [44]. Our data potentially support this, but it also shows that RS was sufficient to increase CORT levels whereas MS was not. The majority of behavioral differences were across sex. Males showed increased escape latency, increased anxiety index, increased EPM total movement, and increased climbing in the FST compared to females. Females showed increased swimming in the FST. There have been mixed reports regarding behavioral profiles following MS [45, 46], but sex differences are well documented [47], especially in the FST [48].
Only one miRNA, miR-26a-3p was significantly associated with the interaction between MS, RS, and sex. In this interaction, MS males showed increased expression of miR-26a compared to control males, while MS did not affect its expression in females. However, in RS animals, MS only increased miR-26a expression in females. miR-26a targets HTR1A, one of the serotonin receptors and is upregulated by antidepressants, fluoxetine and reboxetine [49]; however, this was only tested in males. A few other studies have shown significant changes in miR-26 following stress including increased expression in mouse prefrontal cortex [50] as well as in male rats prefrontal cortex who experienced repeated and ancestral stress [51]. Sex differences following ELS, especially stress sensitivity in males prior to puberty, may result from interactions between stress neurobiology and peripubertal sex hormone changes [52]. Future studies should systematically elucidate sex differences in how timing of stress affect miR-26, among others, and its gene targets such as HTR1A. miR-3593 showed the greatest fold change in RS vs. non-RS animals. Cattaneo, Cattane [53] found that hippocampal miR-3593 was downregulated in a rodent prenatal ELS model, but there are no reports in RS or other acute stress. In MS animals, we tested for an interaction between RS, Enr, and sex. In contrast to the 3-way interaction between MS, RS, and sex, there were several miRNAs significantly affected by the 3-way interaction between RS, Enr, and sex in MS animals. Interestingly, Enr in RS animals caused the opposite fold change direction as 


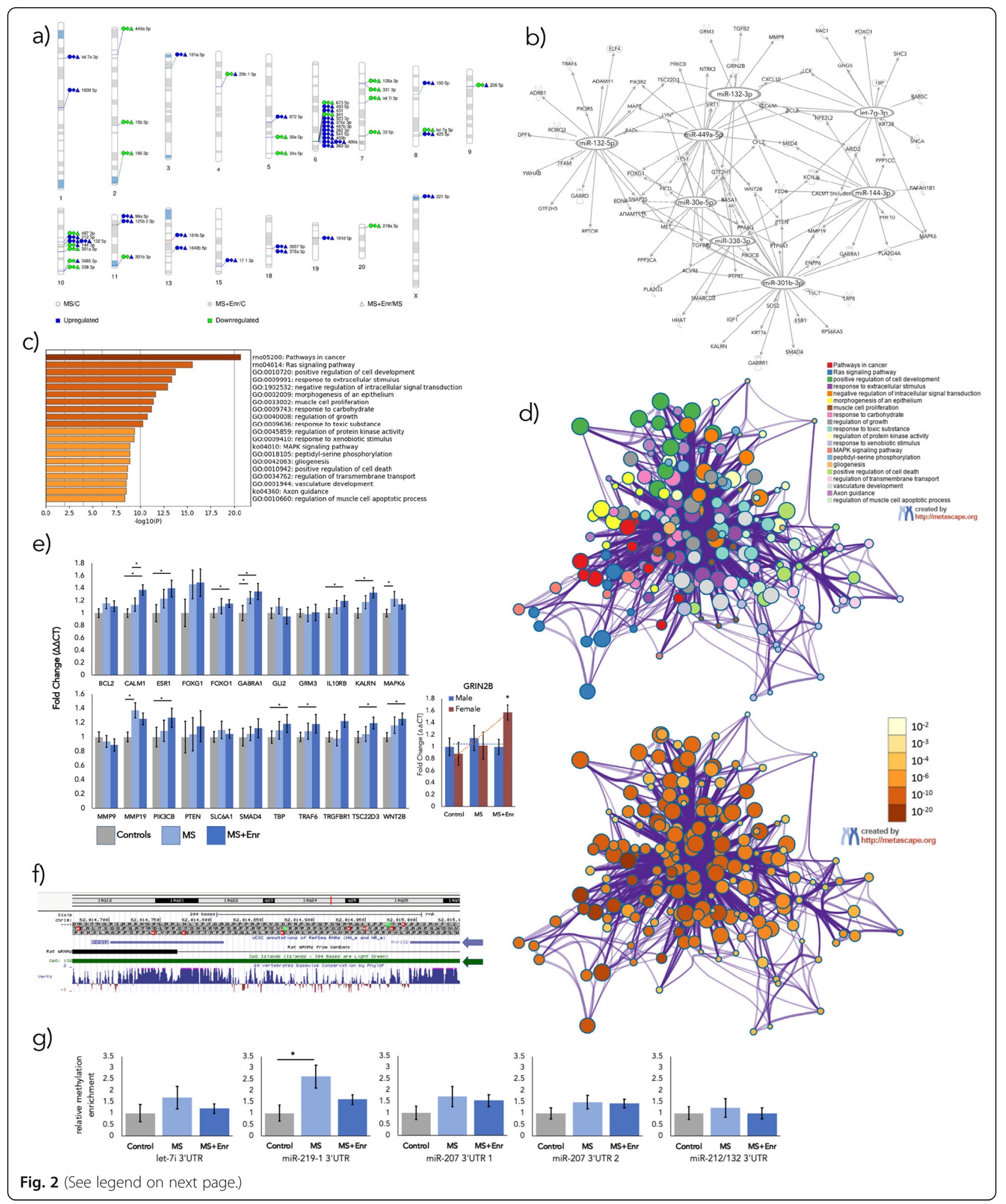




\section{(See figure on previous page.)}

Fig. 2 miRNA, gene targets, and ontologies associated with maternal separation and enrichment. a two sizeable groups of miRNAs altered by MS and Enr localized onto chromosomes 6 and 10. Direction of fold change in MS relative to controls, MS + Enr relative to controls, and MS + Enr relatve to MS are represented as circles, diamonds, and triangles, respectively (blue indicates upregulation and green, downregulation). $\mathbf{b}$ miRNA gene target network shows that 8 significantly altered miRNAs target many stress-related genes and share many targets. c MetascapeGO revealed functions most associated with significant miRNAs' gene targets (Top 3: rno05200-"pathways in cancer", rno04014-"Ras signaling pathway", and GO:0010720-"postive regulation of cell development"; more significant bars are darker colored). $\mathbf{d}$ Metascape also mapped similar ontologies based on common member genes (top map) and significance (bottom map). There is a high level of overlap in membership of these gene targets in Metascape ontologies. e Changes in miRNA gene target expression was tested using qPCR. Bars represent $\mathrm{M} \pm \mathrm{SEM}$ and significance were determined by independent samples t-test ( $n=24$ animals per group) and one-way ANOVA (for GRIN2B). GABRA1, MAPK6, and MMP19 were significantly upregulated by MS $(\mathrm{t}(46)>2.035, p<0.048)$ and MAPK6 and MMP19 were partially recovered by Enr. GRIN2B was the only gene affected by the interaction between sex and group $(F(2,72)=3.703, p=0.030)$. $\mathbf{f} C P G$ islands were identified near miR-promoter region using the UCSC genome browser; the blue arrow shows miR-212 to the left and miR-132 on the right. The row marked in green shows a CPG island. $\mathbf{g}$ methylation in the $3^{\prime} U T R$ of miR-219-1 was significantly increased by MS $(t(30)=2.29, p=0.029)$; due to limited tissue availability, methylation was tested in $n=8$ animals per group (compared to $n=12$ for gene and miRNA expression). For miR-207, methylation in two distinct regions of one CPG island was tested. Significance: ${ }^{*} p<0.05$. Abbreviations: MS-maternal separation, C-controls, Enr-environmental enrichment, UTR-untranslated region, SEM-standard error of the mean

compared to Enr in non-RS animals. For example, Enr alone was associated with a 1.54-fold decrease in miR702-3p expression compared to controls; Enr + RS males showed a 1.24-fold increase in miR-702-3p. This consistently occurred for almost every miRNA. This finding suggests that Enr and RS exhibit opposing miRNA profiles across many miRNAs.

Two large groups of miRNAs associated with MS were colocalized on chromosome 6 and 10. Several significant miRNAs within close proximity could indicate regulation by a common mechanism such as competitive endogenous RNAs [54]. Additionally, when miRNA are closely colocalized they may be transcribed in tandem as a cluster which may indicate similar or dependent functions as well as common regulatory elements [55]. Of the tested gene targets, CALM1 is most closely localized near ( 9500 kilobases) the miRNA cluster on chromosome 6. Another gene, Delta-like noncanonical notch ligand 1 (DLK1), was identified extremely close $(\sim 100$ kilobases) to the chromosome 6 cluster. DLK1 interacts with $\mathrm{NOTCH}$ signaling pathways and is especially involved in pituitary gland development [56]. Furthermore, after adolescence, DLK1 is expressed solely in neuroendocrine tissues in adults. DLK1 and NOTCH signaling changes as mediated by miRNAs may an important avenue for future stress and depression-related studies.

There are previous reports which suggest that estrus phase significantly alters the behavioral response to stress [57]. Our findings showed that only the FST was affected by estrus timing. Proestrus/diestrus (p/d) animals showed decreased swimming, yet increased climbing compared to estrus/metestrus $(\mathrm{e} / \mathrm{m})$ animals. This could be attributed to the increased ratio of MS to control animals in $\mathrm{p} / \mathrm{d}$ group; however, no other behaviors showed significant differences. Kokras, Antoniou [48] also found that animals in the proestrus and diestrus phase exhibited greater climbing duration following treatment with sertraline compared to animals in estrus.
A total of 6 miRNAs showed differential expression between the $\mathrm{p} / \mathrm{d}$ and $\mathrm{e} / \mathrm{m}$ groups. Of these, miRs-362-3p, -374-5p, and - 384-5p are localized on the $\mathrm{X}$ chromosome. MiR-384-5p has been shown to regulate SOX9, a member of the NFkB pathway [58]. SOX 9 is also an embryonic transcription factor which responds to estrogen during sex determination [59]. Our findings, in general, do not support a particularly robust contribution of estrus timing to stress susceptibility.

In order to identify miRNAs and their regulatory pathways specific to MS and Enr, we compared miRNA expression between control (non-MS) animals, MS animals without Enr, and MS + Enr. While there were a greater number of upregulated miRNAs in MS compared to controls, downregulated miRNAs exhibited greater fold change differences between MS and controls. miRs-144$3 p,-206-5 p,-29 b-1-5 p$, and -301b-3p showed the greatest decrease in expression. In an augmented maternal care model using MS, Vogel Ciernia, Laufer [28] reported increased hypothalamic miR-144 expression. Since this model produces increased maternal care behaviors, this could be considered a form of enrichment. However, in comparison, our MS + Enr animals showed similar expression of miR-144 as the MS group. Several miR-29 family miRNAs have been reported in both rodent and human ELS studies. Uchida, Hara [30] found an increase in miR-29a following MS. On the other hand, Cattane, Mora [60] found increased miR-29b-3p and $-29 c-3 p$ in healthy individuals with a history of ELS. In humans, at least, resilient individuals show opposite expression changes compared to our MS animals. Moreover, our findings show that Enr dampened the effect of MS on miR-29b-1-5p expression. miRNA gene target network mapping revealed several miRNA hubs for potential gene regulation. In another study of 180-min MS, miR-132 was upregulated compared to control [30]. We found similar increases in expression in both MS and $\mathrm{MS}+$ Enr groups. In MDD patients, plasma miR-132 
expression is elevated and is also reduced by escitalopram treatment [61]. Let-7 g-3p was another hub miRNA that was significantly downregulated by MS. The let-7 family of miRNAs has been strongly implicated in various aspects of MDD pathophysiology [62]. Between various hub miRNAs, several common gene targets were found including PTEN, MAPK6, CALM1, WNT2B, and GRIN2B.

From the list of most commonly target genes, we selected several genes for qCPR-based validation. GABRA1 (GABA receptor subunit alpha-1) showed increased expression in both MS and MS + Enr groups. MAPK6 (mitogen-activated protein kinase 6) and MMP19 (matrix metalloproteinase-19) showed increased expression in MS, but MS + Enr groups showed expression reversal. One study found that GABRA1 was downregulated in postmortem brain of MDD patients who died by suicide [63]. Our findings only show increased expression of WNT2B in MS + Enr animals compared to controls, though not in MS animals without Enr. Wnt family proteins act on the $\beta$-catenin pathway and may play a role in mood disorder pathophysiology [64]. Our group recently found decreased WNT5B expression associated with increased miR-128 in rodents with learned helplessness [65]. Another study found that neonatal MS reduced myelination in the prefrontal cortex via Wnt signaling changes [66].. MAPK6 encodes ERK3 and is widely expressed in the brain. ERK3 plays an important role in neonatal growth [67]. MAPK pathways have also been found to regulate some MMPs [68]. Wojtowicz and Mozrzymas [69] found that FN-439, a synthetic compound which blocks active MMP sites via chelation of $\mathrm{Zn}^{2+}$, led to a loss of long-term potentiation in CA1 of the hippocampus. However, there is currently little research specific to MMP19.

All 4 of these genes are targeted by miR-301b-3p. Following the canonical pattern of expression between miRNAs and their targets, miR-301b-3p was significantly downregulated while all 4 genes were upregulated. Furthermore, MS + Enr animals showed miR-301b expression more similar controls-the opposite as seen in MAPK6 and MMP19 expression. miR-144 was also a targeting miRNA for both MMP19 and MAPK6. This is one of the first studies to support coregulation of these pathways by miRNAs. Furthermore, they may respond to Enr as a preventative for depressive behavior, particularly anhedonia. In our study, Enr was associated with significant improvement in several behavior. Sucrose preference levels in MS + Enr animals were closer to that of control animals, showing the potential for Enr to protect against the depressogenic effects of MS. Enr animals also showed significantly increased total movement in the EPM indicating a reduced anxiety phenotype. Reduced adrenal weight in Enr animals suggests that Enr reduced engagement of the HPA stress axis, although CORT levels were unchanged.

We identified CPG islands near 4 miRNA promoter regions. miR-219a showed a significant increase in relative methylation following MS. Following the canonical relationship between methylation and decreased gene transcription [70], miR-219 was significantly downregulated in both MS and MS + Enr cohorts. This is the first study to implicate methylation as a potential mechanism of miRNA regulation following MS. TGFBR2 and ESR1 (Estrogen receptor 1) are both gene targets of miR-219a; however, neither showed significant upregulation following MS. A study of environmental enrichment in rats found increased expression of miR-219 in serum exosomes [71]. This study also transfused the serum of young, enriched animals into aging animals and found evidence of increased oligodendrocyte development. Further research is needed to parse the interaction between miRNA expression, miRNA methylation, estrogens, and environmental enrichment.

\section{Conclusions}

Both ELS and acute stress have widespread effects on miRNA expression which may mediate changes in stress-related behaviors. We found that sex significantly altered miRNA expression in response to stress, often with male animals showing more robust and extensive changes. This effect may be due to pre-pubertal timing of MS and highlights the need for more detailed characterization of the effect of ELS timing on behavior and neurobiology. Furthermore, this study identified several hypothalamic miRNAs of interest with regards to ELS. It is important for future studies to test whether direct manipulation of these candidate miRNAs lead to or even prevent later stress susceptibility and whether these behavioral changes are immediate and/or longlasting. Enrichment had a particularly strong effect on animal behavior and miRNA expression and even reversed some of the effects of MS especially in the MAPK signaling pathway. These findings highlight enrichment as a noninvasive means of altering miRNAs which could prove useful in treating or preventing MDD onset. Finally, increased methylation may mediate some of the changes in miRNA expression resulting from ELS. Future studies utilizing next-generation sequencing will be crucial to detecting methylation of miRNAs with better resolution.

\section{Methods}

\section{Animals}

The study was approved by the Institutional Animal Care and Use Committee at the University of Alabama at Birmingham and was performed in accordance with relevant regulations, including ARRIVE guidelines. 
Animals were housed under standard care conditions (ad libitum food and water, $27^{\circ} \mathrm{C}, 12$-h light-dark cycle) for the duration of the experiment. Each group included 6 animals. The expanded methods are described in Supplementary File 1.

\section{Maternal separation}

An overview of the experiment is shown in Fig. 1a. Each litter of Holtzman rats (Envigo, Indianapolis, IN, USA) was randomly assigned to either the control (non-MS) or MS group. Non-MS controls were handled for $5 \mathrm{~min}$ from PND 1-14. MS pups were separated from the dam and housed individually on a $33^{\circ} \mathrm{C}$ heating pad for 180 min each morning until PND 14. Pups were weaned from the dam on PND 21.

\section{Restraint stress}

Animals from both control and MS groups were randomly assigned to RS or control groups (non-RS). At PND 80, RS animals were placed in $20 \mathrm{~cm}$ restrainer tubes for $120 \mathrm{~min}$ each day for 7 days. Non-RS controls were handled but not restrained.

\section{Environmental enrichment}

A subset of MS and MS + RS animals was randomly assigned to receive Enr from PND 21 to 90. Enrichment included colored toys, tubes, shreddable cotton and paper objects, and manzanita wood which were rotated weekly to maintain novelty. Non-enriched animals were housed conventionally.

\section{Animal behavior}

\section{Sucrose preference test}

Sucrose preference was tested immediately following RS to assess anhedonia as previously described [72]. Briefly, on day 1 , the animals were given sucrose $1 \%(w / v)$. After acclimation on day 2 , the animals were given access to both regular water and sucrose. Then the animals fasted for $24 \mathrm{~h}$. On day 4 , the animals were housed individually and given a premeasured $500 \mathrm{~mL}$ bottle of $1 \%$ sucrose and regular water. After $8 \mathrm{~h}$, each of the bottles was measured for consumption and sucrose preference was calculated as previously described [72].

\section{Elevated plus maze}

Animals were placed individually in the center of a raised plus-shaped platform $(50 \times 50 \mathrm{~cm})$ with two open and two walled arms ( $15 \mathrm{~cm}$ tall, open roof). For $5 \mathrm{~min}$, the animals were recorded using Noldus Ethovision XT 11.5. Open and closed arm time and frequency were recorded and anxiety index was calculated as published earlier [73].

\section{Forced swim test}

Animals were acclimated for 15-min to an acrylic cylinder $(28 \mathrm{~cm}$ diameter $\times 46 \mathrm{~cm}$ tall) filled to $25 \mathrm{~cm}$ with room temperature water. $24 \mathrm{~h}$ later, each animal was recorded in the same swim condition for $6 \mathrm{~min}$ and was returned to their home cage. A rater blinded to the conditions scored the videos using Kinoscope [74].

\section{Shuttle escape test}

As reported previously [72], escape latency was tested using a two-chamber shuttlebox with an electrified grid floor $(70 \mathrm{~cm} \times 20 \mathrm{~cm} \times 20 \mathrm{~cm}$, Med Associates, IN, USA). For the initial 5 trials, foot shocks were delivered on a variable interval schedule $(0.6 \mathrm{~mA}, 60 \mathrm{~s}$ average interval) and terminated when the animal crossed into the opposite chamber. For the next 25 trials, the foot shocks terminated after the animal crossed into the opposite chamber and back. Escape latency was recorded by a PC connected to the shuttle box and shock generator.

\section{Vaginal cytology, tissue collection, and RNA isolation}

The morning following escape testing, prior to tissue collection, female animals were tested for estrous phase by vaginal lavage. A blinded-rater assessed the samples according to cell morphology as previously described $[75,76]$. The animals were anesthetized with isofluorane and blood was collected via cardiac puncture. The brain and adrenal glands were dissected, flash frozen, and stored at $-80^{\circ} \mathrm{C}$ until further use. RNA was extracted from hypothalamus using TRIzol (Invitrogen, NY, USA) as described in Roy, Dunbar [34]. RNA concentration and quality were tested using Nanodrop 2000C Spectrophotometer; $260 / 280 \mathrm{~nm}>1.7$ was considered pure.

\section{Corticosterone, estradiol, and progesterone ELISAs}

CORT, Estradiol, and Progesterone were quantified in platelet-free plasma using enzyme-linked immunosorbent assay (ELISA) (Enzo Life Sciences, NY, USA).

\section{miRNA sequencing}

RNA sequencing libraries were prepared using the Qiaseq miRNA library kit (Qiagen, Hilden, Germany) and were sequenced on a NextSeq 500 (Illumina, CA, USA). Data were extracted using Qiagen Gene Globe and CPM was calculated using edgeR.

\section{Statistical approach}

A $2 \times 2 \times 2$ ANOVA was conducted in $R$ to examine behavior and miRNA expression differences between MS and RS groups as well as sex. We also conducted a $2 \times 2$ $\times 2$ ANOVA to examine the interaction of RS, sex, and Enr within MS animals on behavior and miRNA expression. Figure $1 \mathrm{~b}$ is a visual representation of the animals/ groups which were used in each analysis. We used a 1- 
way ANOVA to compare control, MS, and MS + Enr group behavior and miRNA expression. Subsequent bioinformatic analysis and follow-up gene expression and methylation studies were based on miRNA expression changes found when comparing control, MS, and MS + enrichment groups. To determine if sex played a role in MS and Enr-related gene target expression, we conducted a 2-way ANOVA. Only 1 gene showed a significant interaction, so the remaining gene expression comparisons were based on pairwise t-tests across group (control, MS, or MS + Enr). Group differences in qPCRbased methylation were tested using pairwise $\mathrm{t}$-tests.

\section{Bioinformatic analysis}

\section{Chromosomal localization}

miRbase.org was used to identify the chromosomal loci for significantly altered miRNAs. The Phenogram application (Ritchie Lab, University of Pennsylvania, USA) was used to plot the location of miRNAs across the rat karyotype.

\section{Gene target prediction, miRNA-gene target networks, and gene ontology}

A primary aim was to determine how MS leads to depression-like behavior via miRNA-based gene regulation. Thus, IPA was used to identify validated and highly predicted gene targets of miRNAs which were significantly altered by MS. This gene-target list was filtered based on each gene's membership in canonical stressrelated pathways. Futhermore, in order to identify a set of miRNAs with the greatest potential impact across these pathways only those pathways with $>16$ gene targets were included. Because miRNAs can target many different genes and each gene may also be targeted by many miRNAs [26], pathways targeted by fewer miRNAs and with fewer compatible gene targets are less likely to be functionally affected by changes in an individual miRNA's expression; it is possible that changes in a single miRNA may be negated by the activity of other miRNAs or even other regulatory mechanism. However, when several miRNAs targeting many genes within the same molecular pathway are altered, it is less likely that this cumulative effect will be countered by another miRNA or other mechanism. The identified miRNA targets were narrowed to 99 genes involved in Axonal Guidance Signaling (27 genes), Glucocorticoid Receptor Signaling (27 genes), Neuroinflammation Signaling (26 genes), Synaptogenesis Signaling (23 genes), Estrogen Receptor Signaling (23 genes), Protein Kinase A Signaling (19 genes), AMPK Signaling (18 genes), and ERK/MAPK Signaling (17 genes). miRNA-gene target networks were created using these 99 genes and significantly altered miRNAs based on one-way ANOVA. Among these miRNA-gene targeting relationships, miRNAs with more than 10 gene targets were identified as gene regulatory hubs and were also visualized in IPA as a miRNA-gene network. ShinyGO v0.61 [77] was used to explore GO associated with miRNA target genes. Metascape [78] was also used to confirm our $\mathrm{GO}$ analysis and to cluster ontologies with similar gene components.

Gene targets for qPCR follow-up were selected from the miRNA hub-gene target network based on their number of miRNA targets. Gene targets with the highest fold change as well as those which showed reversal of expression changes after Enr were also selected for qPCR-based expression testing.

\section{CDNA synthesis and gene target expression by qPCR}

$1 \mu \mathrm{g}$ of RNA was reverse transcribed using an oligo dT priming method to synthesize first strand complimentary DNA (cDNA). Relative gene abundance was tested using qPCR and BrightGreen chemistry (Applied Biological Material, Canada). Primer sequences for the selected genes are listed in Supplementary Table 4. Livak's $\Delta \Delta$ CT [79] was method to quantify group differences. The data was normalized to the geometric mean of GAPDH, $B$-actin, and 18srRNA. Because there was little variability in the housekeeping genes, each sample was tested individually for each gene of interest.

\section{Methylated DNA immunoprecipitation (MeDIP) and miRNA promoter region methylation by qPCR}

Using the UCSC rodent genome browser tool, we searched for CPG islands near the promoter region of significantly altered miRNAs. We only found CPG islands upstream of 5 miRNAs and designed primers (Supplementary Table 4) to target these regions using Primer3 (v0.04.0; Untergasser, Cutcutache [80]). Genomic DNA (gDNA) was isolated from hypothalamus using phenol: chloroform: isoamylalcohol $(25: 24: 1 \mathrm{~V} / \mathrm{V})$ chemistry. gDNA was sheared and immunoprecipitated with 5-methyl cytosine antibody (Zymo Research, CA). BrighGreen-based qPCR was used to quantify relative methylation enrichment in the immunoprecipitated DNA using the designed primers. Because of limited tissue availability, miRNA promoter methylation was tested in 48 of 72 original samples ( $n=4$ per group, including males and females).

\section{Abbreviations}

ELS: early life stress; MDD: major depressive disorder; MS: maternal separation; RS: restraint stress; HPA : hypothalamic pituitary adrenal; $\mathrm{CRH}$ : corticotropin releasing hormone; $\mathrm{ACTH}$ : adrenocorticotropin releasing hormone; CORT: corticosterone; miRNA, miR: microRNA; RISC: RNA-induced silencing complex; Enr: environmental enrichment; EPM : elevated plus maze; FST: forced swim test; $\mathrm{p} / \mathrm{d}$ : proestrus/diestrus; e/m: estrus/metestrus; CPM: counts per million; GO: gene ontology; cDNA: complimentary DNA: MeDIP: methylated DNA immunoprecipitation 


\section{Supplementary Information}

The online version contains supplementary material available at https://doi. org/10.1186/s12864-021-08003-4

\section{Additional file 1.}

\section{Acknowledgements}

We would like to thank Dr. Bhaskar Roy for his help in molecular studies. Kevin Prall, Emma Jones, and Grant Schell helped with behavior data and animal tissue collection. We would also like to acknowledge Subhan Ahmed and Genelle Samson for their blind ratings of behavior data. miRNA sequencing for this study was conducted by UAB's Heflin Genomics Core (Birmingham, AL, USA).

\section{Authors' contributions}

LAM contributed to the study design, data collection, formal analysis, data curation and wrote the original draft. YD conceptualized the project and methodology, provided resources, and reviewed and edited the final draft.

\section{Funding}

The research was partly supported by grants from National Institute of Mental Health (MH082802; MH101890; MH100616; MH107183; MH112014; MH118884) and American Foundation for Suicide Prevention (DIG-0-041-18) to Dr. Dwivedi. The funding agency had no input on the study design, collection, analysis, or interpretation of the data.

\section{Availability of data and materials}

The datasets generated and analyzed during the current study are available in the NCBI Sequence Read Archive (SRA) repository, at http://www.ncbi.nlm. nih.gov/bioproject/728805 and accession \#: PRJNA728805.

\section{Declarations}

\section{Ethics approval and consent to participate}

Animal experiments as a part of this study were approved by the Institutional Animal Care and Use Committee at the University of Alabama at Birmingham (\#20086). All experiments were carried out in accordance with relevant regulations and guidelines. The ARRIVE guidelines were followed throughout the study.

\section{Consent for publication}

Not applicable.

\section{Competing interests}

The authors declare that they have no competing interests.

Received: 20 April 2021 Accepted: 13 September 2021

Published online: 28 September 2021

\section{References}

1. Middlebrooks JS, Audage NC. The effects of childhood stress on health across the lifespan. Atlanta: Centers for Disease Control and Prevention, National Center for Injury Prevention and Control. 2008. p. 3-16.

2. Greenberg PE, Fournier A-A, Sisitsky T, Pike CT, Kessler RC. The economic burden of adults with major depressive disorder in the United States (2005 and 2010). J Clin Psychiatry. 2015;76(2):155-62. https://doi.org/10.4088/ JCP. 14 m09298.

3. Kendler KS, Karkowski LM, Prescott CA. Causal relationship between stressful life events and the onset of major depression. Am J Psychiatry. 1999;156(6): 837-41. https://doi.org/10.1176/ajp.156.6.837.

4. Seok BJ, Jeon S, Lee J, Cho SJ, Lee YJ, Kim SJ. Effects of early trauma and recent stressors on depression, anxiety, and anger. Front Psychiatry. 2020;11: 744. https://doi.org/10.3389/fpsyt.2020.00744.

5. Daskalakis NP, Bagot RC, Parker KJ, Vinkers CH, de Kloet ER. The three-hit concept of vulnerability and resilience: toward understanding adaptation to early-life adversity outcome. Psychoneuroendocrinology. 2013;38(9):1858-73. https://doi.org/10.1016/.jpsyneuen.2013.06.008.

6. McEwen BS, Gray JD, Nasca C. 60 YEARS OF NEUROENDOCRINOLOGY: redefining neuroendocrinology: stress, sex and cognitive and emotional regulation. J Endocrinol. 2015;226(2):T67-83. https://doi.org/10.1530/JOE-150121

7. U.S. Department of Health \& Human Services, Administration for Children and Families, Administration on Children, Youth and Families, Children's Bureau. Child maltreatment 2016. 2018. Available from https://www.acf.hhs. gov/cb/research-data-technology/statistics-research/child-maltreatment.

8. CfBHSa Q. 2016 National Survey on drug use and health: methodological summary and definitions. Substance Abuse and Mental Health Services Administration: Rockville, MD; 2017.

9. Coiro P, Pollak DD. Sex and gender bias in the experimental neurosciences: the case of the maternal immune activation model. Transl Psychiatry. 2019; 9(1):90. https://doi.org/10.1038/541398-019-0423-8.

10. Heim C, Newport DJ, Heit S, Graham YP, Wilcox M, Bonsall R, et al. Pituitaryadrenal and autonomic responses to stress in women after sexual and physical abuse in childhood. Jama. 2000;284(5):592-7. https://doi.org/10.1 001/jama.284.5.592.

11. Vazquez DM, Lopez JF, Van Hoers H, Watson SJ, Levine S. Maternal deprivation regulates serotonin $1 \mathrm{~A}$ and $2 \mathrm{~A}$ receptors in the infant rat. Brain Res. 2000;855(1):76-82. https://doi.org/10.1016/S0006-8993(99)02307-0.

12. Horii-Hayashi N, Sasagawa T, Matsunaga W, Matsusue Y, Azuma C, Nishi M. Developmental changes in desensitisation of c-Fos expression induced by repeated maternal separation in pre-weaned mice. J Neuroendocrinol. 2013; 25(2):158-67. https://doi.org/10.1111/j.1365-2826.2012.02377.x.

13. Price JL. Comparative aspects of amygdala connectivity. Ann N Y Acad Sci. 2003;985(1):50-8. https://doi.org/10.1111/j.1749-6632.2003.tb07070.x.

14. Öngür D, Price JL. The Organization of Networks within the orbital and medial prefrontal cortex of rats. Cereb Cortex: Monkeys and Humans; 2000.

15. Price JL, Drevets WC. Neurocircuitry of mood disorders. Neuropsychopharmacology : Official Publication of the American College of Neuropsychopharmacology. 2010;35(1):192-216. https://doi.org/10.1038/ npp.2009.104

16. Sakurai T. The role of orexin in motivated behaviours. Nat Rev Neurosci. 2014;15(11):719-31. https://doi.org/10.1038/nrn3837.

17. Martinez-Hernandez J, Lanuza E, Martinez-Garcia F. Selective dopaminergic lesions of the ventral tegmental area impair preference for sucrose but not for male sexual pheromones in female mice. Eur J Neurosci. 2006;24(3):88593. https://doi.org/10.1111/j.1460-9568.2006.04944.x.

18. Shekhar A. GABA receptors in the region of the dorsomedial hypothalamus of rats regulate anxiety in the elevated plus-maze test. I Behav Meas Brain Res. 1993;627(1):9-16. https://doi.org/10.1016/0006-8993(93)90742-6.

19. Bidzinski A, Jankowska E, Pucilowski O. Antidepressant-like action of nicardipine, verapamil and hemicholinium-3 injected into the anterior hypothalamus in the rat forced swim test. Pharmacol Biochem Behav. 1990; 36(4):795-8. https://doi.org/10.1016/0091-3057(90)90079-W.

20. Heim C, Nemeroff CB. The role of childhood trauma in the neurobiology of mood and anxiety disorders: preclinical and clinical studies. Biol Psychiatry. 2001;49(12):1023-39. https://doi.org/10.1016/S0006-3223(01)01157-X.

21. Heim C, Newport DJ, Mletzko T, Miller AH, Nemeroff CB. The link between childhood trauma and depression: insights from HPA axis studies in humans. Psychoneuroendocrinology. 2008;33(6):693-710. https://doi.org/1 0.1016/.jpsyneuen.2008.03.008.

22. Dwivedi Y. microRNA-124: a putative therapeutic target and biomarker for major depression. Expert Opin Ther Targets. 2017;21 (7):653-6. https://doi. org/10.1080/14728222.2017.1328501.

23. O'Connor RM, Dinan TG, Cryan JF. Little things on which happiness depends: microRNAs as novel therapeutic targets for the treatment of anxiety and depression. Mol Psychiatry. 2012;17(4):359-76. https://doi.org/1 0.1038/mp.2011.162

24. Dwivedi Y. Emerging role of microRNAs in major depressive disorder: diagnosis and therapeutic implications. Dialogues Clin Neurosci. 2014;16(1): 43-61. https://doi.org/10.31887/DCNS.2014.16.1/ydwivedi.

25. Roy B, Yoshino Y, Allen L, Prall K, Schell G, Dwivedi Y. Exploiting circulating MicroRNAs as biomarkers in psychiatric disorders. Mol Diagn Ther. 2020; 24(3):279-98. https://doi.org/10.1007/s40291-020-00464-9.

26. Bartel DP. Metazoan MicroRNAs. Cell. 2018;173(1):20-51. https://doi.org/10.1 016/j.cell.2018.03.006.

27. Allen L, Dwivedi Y. MicroRNA mediators of early life stress vulnerability to depression and suicidal behavior. Mol Psychiatry. 2020;25(2):308-20. https:// doi.org/10.1038/s41380-019-0597-8.

28. Vogel Ciernia A, Laufer BI, Dunaway KW, Mordaunt CE, Coulson RL, Totah TS, et al. Experience-dependent neuroplasticity of the developing 
hypothalamus: integrative epigenomic approaches. Epigenetics. 2018;13(3): 318-30. https://doi.org/10.1080/15592294.2018.1451720.

29. Vetulani J. Early maternal separation: a rodent model of depression and a prevailing human condition. Pharmacol Rep. 2013;65(6):1451-61. https://doi. org/10.1016/S1734-1140(13)71505-6.

30. Uchida S, Hara K, Kobayashi A, Funato H, Hobara T, Otsuki K, et al. Early life stress enhances behavioral vulnerability to stress through the activation of REST4-mediated gene transcription in the medial prefrontal cortex of rodents. J Neurosci. 2010;30(45):15007-18. https://doi.org/10.1523/ JNEUROSCI.1436-10.2010.

31. Bahi A. Sustained lentiviral-mediated overexpression of microRNA124a in the dentate gyrus exacerbates anxiety- and autism-like behaviors associated with neonatal isolation in rats. Behav Brain Res. 2016;311:298-308. https:// doi.org/10.1016/j.bbr.2016.05.033.

32. Parodi F, Carosio R, Ragusa M, Di Pietro C, Maugeri M, Barbagallo D, et al. Epigenetic dysregulation in neuroblastoma: a tale of miRNAs and DNA methylation. Biochim Biophys Acta. 2016;1859(12):1502-14. https://doi.org/1 0.1016/j.bbagrm.2016.10.006.

33. Bostrom AE, Ciuculete DM, Attwood M, Krattinger R, Nikontovic L, Titova OE, et al. A MIR4646 associated methylation locus is hypomethylated in adolescent depression. J Affect Disord. 2017;220:117-28. https://doi.org/10.1 016/j.jad.2017.05.017

34. Roy B, Dunbar M, Shelton RC, Dwivedi Y. Identification of microRNA-124-3p as a putative epigenetic signature of major depressive disorder. Neuropsychopharmacology : Official Publication of the American College of Neuropsychopharmacology. 2017;42(4):864-75. https://doi.org/10.1038/ npp.2016.175.

35. Wortzel JR, Turner BE, Weeks BT, Fragassi C, Ramos V, Truong T, et al. Trends in mental health clinical research: Characterizing the ClinicalTrials.gov registry from 2007-2018. PLoS ONE. 2020;15(6):e0233996.

36. Kaplan MJ, Klinetob NA. Childhood emotional trauma and chronic posttraumatic stress disorder in adult outpatients with treatment-resistant depression. J Nerv Ment Dis. 2000;188(9):596-601. https://doi.org/10.1097/ 00005053-200009000-00006.

37. Durairaj RV, Koilmani ER. Environmental enrichment modulates glucocorticoid receptor expression and reduces anxiety in Indian field male mouse Mus booduga through up-regulation of microRNA-124a. Gen Comp Endocrinol. 2014;199:26-32. https://doi.org/10.1016/j.ygcen.2014.01.005.

38. Sztainberg Y, Kuperman Y, Tsoory M, Lebow M, Chen A. The anxiolytic effect of environmental enrichment is mediated via amygdalar CRF receptor type 1. Mol Psychiatry. 2010;15(9):905-17. https://doi.org/10.103 8/mp.2009.151.

39. Brenes Saenz JC, Villagra OR, Fornaguera TJ. Factor analysis of forced swimming test, sucrose preference test and open field test on enriched, social and isolated reared rats. Behav Brain Res. 2006;169(1):57-65. https:// doi.org/10.1016/j.bbr.2005.12.001.

40. Morley-Fletcher S, Rea M, Maccari S, Laviola G. Environmental enrichment during adolescence reverses the effects of prenatal stress on play behaviour and HPA axis reactivity in rats. Eur J Neurosci. 2003;18(12):3367-74. https:// doi.org/10.1111/j.1460-9568.2003.03070.x.

41. Weinstock M. Does Prenata stress impair coping and regulation of hypothalamic-pituitary-adrenal Axis? Neurosci Biobehav Rev. 1997;21(1):110. https://doi.org/10.1016/S0149-7634(96)00014-0.

42. Smalheiser NR, Lugli G, Rizavi HS, Zhang H, Torvik VI, Pandey GN, et al. MicroRNA expression in rat brain exposed to repeated inescapable shock: differential alterations in learned helplessness vs. non-learned helplessness. Int J Neuropsychopharmacol. 2011;14(10):1315-25. https://doi.org/10.1017/ S1461145710001628.

43. Walf AA, Frye CA. The use of the elevated plus maze as an assay of anxietyrelated behavior in rodents. Nat Protoc. 2007;2(2):322-8. https://doi.org/10.1 038/nprot.2007.44

44. Barha CK, Pawluski JL, Galea LA. Maternal care affects male and female offspring working memory and stress reactivity. Physiol Behav. 2007;92(5): 939-50. https://doi.org/10.1016/j.physbeh.2007.06.022.

45. Murthy S, Gould E. Early life stress in rodents: animal models of illness or resilience? Front Behav Neurosci. 2018;12:157. https://doi.org/10.3389/ fnbeh.2018.00157.

46. Wang D, Levine JLS, Avila-Quintero V, Bloch M, Kaffman A. Systematic review and meta-analysis: effects of maternal separation on anxiety-like behavior in rodents. Transl Psychiatry. 2020;10(1):174. https://doi.org/10.103 8/s41398-020-0856-0.
47. Kokras N, Dalla C. Sex differences in animal models of psychiatric disorders. Br J Pharmacol. 2014;171(20):4595-619. https://doi.org/10.1111/bph.12710.

48. Kokras N, Antoniou K, Mikail HG, Kafetzopoulos V, Papadopoulou-Daifoti Z, Dalla C. Forced swim test: what about females? Neuropharmacology. 2015; 99:408-21. https://doi.org/10.1016/j.neuropharm.2015.03.016.

49. Xie L, Chen J, Ding YM, Gui XW, Wu LX, Tian S, et al. MicroRNA-26a-2 maintains stress resiliency and antidepressant efficacy by targeting the serotonergic autoreceptor HTR1A. Biochem Biophys Res Commun. 2019; 511(2):440-6. https://doi.org/10.1016/j.bbrc.2019.02.078.

50. Rinaldi A, Vincenti S, De Vito F, Bozzoni I, Oliverio A, Presutti C, et al. Stress induces region specific alterations in microRNAs expression in mice. Behav Brain Res. 2010;208(1):265-9. https://doi.org/10.1016/j.bbr.2009.11.012.

51. Ambeskovic M, Babenko O, Inytskyy Y, Kovalchuk I, Kolb B, Metz GAS. Ancestral stress alters lifetime mental health trajectories and cortical Neuromorphology via epigenetic regulation. Sci Rep. 2019;9(1):6389. https:// doi.org/10.1038/s41598-019-42691-z.

52. Bale TL, Epperson CN. Sex differences and stress across the lifespan. Nat Neurosci. 2015;18(10):1413-20. https://doi.org/10.1038/nn.4112.

53. Cattaneo A, Cattane N, Malpighi C, Czamara D, Suarez A, Mariani N, et al. FoxO1, A2M, and TGF-beta1: three novel genes predicting depression in gene $X$ environment interactions are identified using cross-species and cross-tissues transcriptomic and miRNomic analyses. Mol Psychiatry. 2018; 23(11):2192-208. https://doi.org/10.1038/s41380-017-0002-4.

54. Bak RO, Mikkelsen JG. miRNA sponges: soaking up miRNAs for regulation of gene expression. Wiley Interdiscip Rev RNA. 2014;5(3):317-33. https://doi. org/10.1002/wrna.1213.

55. Lee $\mathrm{Y}$, Jeon K, Lee JT, Kim S, Kim VN. MicroRNA maturation: stepwise processing and subcellular localization. EMBO J. 2002;21(17):4663-70. https://doi.org/10.1093/emboj/cdf476.

56. Falix FA, Aronson DC, Lamers WH, Gaemers IC. Possible roles of DLK1 in the notch pathway during development and disease. Biochim Biophys Acta. 2012;1822(6):988-95. https://doi.org/10.1016/j.bbadis.2012.02.003.

57. Jaric I, Rocks D, Cham H, Herchek A, Kundakovic M. Sex and estrous cycle effects on anxiety- and depression-related phenotypes in a two-hit developmental stress model. Front Mol Neurosci. 2019;12:74. https://doi. org/10.3389/fnmol.2019.00074

58. Zhang W, Cheng P, Hu W, Yin W, Guo F, Chen A, et al. Inhibition of microRNA-384-5p alleviates osteoarthritis through its effects on inhibiting apoptosis of cartilage cells via the NF-kappaB signaling pathway by targeting SOX9. Cancer Gene Ther. 2018;25(11-12):326-38. https://doi.org/1 0.1038/s41417-018-0029-y.

59. Mork L, Capel B. Oestrogen shuts the door on SOX9. BMC Biol. 2010;8(1): 110. https://doi.org/10.1186/1741-7007-8-110.

60. Cattane N, Mora C, Lopizzo N, Borsini A, Maj C, Pedrini L, et al. Identification of a miRNAs signature associated with exposure to stress early in life and enhanced vulnerability for schizophrenia: new insights for the key role of miR-125b-1-3p in neurodevelopmental processes. Schizophr Res. 2018;205: 63-75. https://doi.org/10.1016/j.schres.2018.07.030.

61. Fang $Y$, Qiu Q, Zhang S, Sun L, Li G, Xiao S, et al. Changes in miRNA-132 and miR-124 levels in non-treated and citalopram-treated patients with depression. J Affect Disord. 2018;227:745-51. https://doi.org/10.1016/j.jad.2 017.11.090.

62. Gururajan A, Naughton ME, Scott KA, O'Connor RM, Moloney G, Clarke G, et al. MicroRNAs as biomarkers for major depression: a role for let-7b and let-7c. Transl Psychiatry. 2016;6(8):e862. https://doi.org/10.1038/tp.2016.131.

63. Sequeira A, Klempan T, Canetti L, ffrench-Mullen J, Benkelfat C, Rouleau GA, et al. Patterns of gene expression in the limbic system of suicides with and without major depression. Mol Psychiatry. 2007;12(7):640-55. https://doi. org/10.1038/sj.mp.4001969.

64. Sani G, Napoletano F, Forte AM, Kotzalidis GD, Panaccione I, Porfiri GM, et al. The wnt pathway in mood disorders. Curr Neuropharmacol. 2012;10(3):23953. https://doi.org/10.2174/157015912803217279.

65. Roy B, Dunbar M, Agrawal J, Allen L, Dwivedi Y. Amygdala-based altered miRNome and epigenetic contribution of miR-128-3p in conferring susceptibility to depression-like behavior via Wnt signaling. Int J Neuropsychopharmacol. 2020;23(3):165-77. https://doi.org/10.1093/ijnp/ pyz071.

66. Yang $Y$, Cheng $Z$, Tang $H$, Jiao $H$, Sun $X$, Cui Q, et al. Neonatal maternal separation impairs prefrontal cortical myelination and cognitive functions in rats through activation of Wnt signaling. Cereb Cortex. 2017;27(5):2871-84. https://doi.org/10.1093/cercor/bhw121. 
67. Klinger S, Turgeon B, Levesque K, Wood GA, Aagaard-Tillery KM, Meloche S. Loss of Erk3 function in mice leads to intrauterine growth restriction, pulmonary immaturity, and neonatal lethality. Proc Natl Acad Sci U S A. 2009;106(39):16710-5. https://doi.org/10.1073/pnas.0900919106.

68. Reunanen N, Li SP, Ahonen M, Foschi M, Han J, Kahari VM. Activation of p38 alpha MAPK enhances collagenase-1 (matrix metalloproteinase (MMP)-1) and stromelysin-1 (MMP-3) expression by mRNA stabilization. J Biol Chem. 2002;277(35):32360-8. https://doi.org/10.1074/jbc.M204296200.

69. Wojtowicz T, Mozrzymas JW. Late phase of long-term potentiation in the mossy fiber-CA3 hippocampal pathway is critically dependent on metalloproteinases activity. Hippocampus. 2010;20(8):917-21. https://doi. org/10.1002/hipo.20787.

70. Kass SU, Pruss D, Wolffe A. How does DNA methylation repress transcription? Trends Genet. 1997;13(11):444-9. https://doi.org/10.1016/501 68-9525(97)01268-7.

71. Pusic AD, Kraig RP. Youth and environmental enrichment generate serum exosomes containing miR-219 that promote CNS myelination. Glia. 2014; 62(2):284-99. https://doi.org/10.1002/glia.22606.

72. Timberlake li M, Roy B, Dwivedi Y. A novel animal model for studying depression featuring the induction of the unfolded protein response in hippocampus. Mol Neurobiol. 2019;56(12):8524-36. https://doi.org/10.1007/ s12035-019-01687-6.

73. Cohen H, Matar MA, Joseph Z. Animal models of post-traumatic stress disorder. Curr Protocols Neurosci. 2013;Chapter 9:Unit 945.

74. Kokras N, Baltas D, Theocharis F, Dalla C. Kinoscope: an open-source computer program for behavioral pharmacologists. Front Behav Neurosci. 2017;11:88. https://doi.org/10.3389/fnbeh.2017.00088.

75. McLean AC, Valenzuela N, Fai S, Bennett SA. Performing vaginal lavage, crystal violet staining, and vaginal cytological evaluation for mouse estrous cycle staging identification. J Vis Exp. 2012;67(67):e4389. https://doi.org/10.3 791/4389.

76. Marcondes FK, Bianchi FJ, Tanno AP. Determination of the estrous cycle phases of rats: some helpful considerations. Braz J Biol. 2002;62(4A):609-14. https://doi.org/10.1590/S1519-69842002000400008.

77. Ge SX, Jung D, Yao R. ShinyGO: a graphical gene-set enrichment tool for animals and plants. Bioinformatics. 2020;36(8):2628-9. https://doi.org/10.1 093/bioinformatics/btz931.

78. Zhou Y, Zhou B, Pache L, Chang M, Khodabakhshi AH, Tanaseichuk O, et al. Metascape provides a biologist-oriented resource for the analysis of systems-level datasets. Nat Commun. 2019;10(1):1523. https://doi.org/10.103 8/s41467-019-09234-6.

79. Livak KJ, Schmittgen TD. Analysis of relative gene expression data using real-time quantitative PCR and the 2(-Delta Delta C(T)) method. Methods. 2001;25(4):402-8. https://doi.org/10.1006/meth.2001.1262.

80. Untergasser A, Cutcutache I, Koressaar T, Ye J, Faircloth BC, Remm M, et al. Primer3--new capabilities and interfaces. Nucleic Acids Res. 2012;40(15):e115. https://doi.org/10.1093/nar/gks596.

\section{Publisher's Note}

Springer Nature remains neutral with regard to jurisdictional claims in published maps and institutional affiliations.

Ready to submit your research? Choose BMC and benefit from:

- fast, convenient online submission

- thorough peer review by experienced researchers in your field

- rapid publication on acceptance

- support for research data, including large and complex data types

- gold Open Access which fosters wider collaboration and increased citations

- maximum visibility for your research: over $100 \mathrm{M}$ website views per year

At BMC, research is always in progress.

Learn more biomedcentral.com/submissions 